\title{
Cross-Species Analysis Defines the Conservation of Anatomically-Segregated VMH Neuron Populations
}

\author{
Alison H. Affinati ${ }^{1}$, Paul V. Sabatini ${ }^{1}$, Cadence True ${ }^{2}$, Abigail J. Tomlinson ${ }^{1}$, Melissa \\ Kirigiti ${ }^{2}$, Sarah R. Lindsley², Chien Li ${ }^{3}$, David P. Olson ${ }^{4}$, Paul Kievit ${ }^{2}$, \\ Martin G. Myers, Jr. ${ }^{1 * *}$, and Alan C. Rupp ${ }^{1 * *}$
}

${ }^{1}$ Department of Internal Medicine, University of Michigan, Ann Arbor, MI, USA.

${ }^{2}$ Oregon National Primate Research Center, Beaverton, OR, USA.

${ }^{3}$ Novo Nordisk Research Center, Seattle, WA, USA.

${ }^{4}$ Department of Pediatrics, University of Michigan, Ann Arbor, MI, USA

${ }^{* *}$ Correspondence:

Alan C. Rupp, PhD

Martin G Myers, Jr., MD, PhD

Department of Internal Medicine

University of Michigan

2800 Plymouth Rd., Bldg 20

Ann Arbor, Ml 48109

Phone: 734-647-9515

Email: ruppa@umich.edu, mgmyers@umich.edu

Conflict of Interest Statement: $C L$ is an employee of Novo Nordisk A/S; the authors have no other competing interests relevant to this manuscript. 


\section{ABSTRACT}

The ventromedial hypothalamic nucleus $(\mathrm{VMH})$ controls diverse behaviors and physiologic functions, suggesting the existence of multiple VMH neural subtypes with distinct functions. Combing Translating Ribosome Affinity Purification with RNA sequencing (TRAP-seq) data with snRNA-seq data, we identified 24 mouse VMH neuron clusters. Further analysis, including snRNA-seq data from macaque tissue, defined a more tractable $\mathrm{VMH}$ parceling scheme consisting of 6 major genetically- and anatomically-differentiated $\mathrm{VMH}$ neuron classes with good cross-species conservation. In addition to two major ventrolateral classes, we identified three distinct classes of dorsomedial VMH neurons. Consistent with previously-suggested unique roles for leptin receptor (Lepr)-expressing VMH neurons, Lepr expression marked a single dorsomedial class. We also identified a class of glutamatergic $\mathrm{VMH}$ neurons that resides in the tuberal region, anterolateral to the neuroanatomical core of the $\mathrm{VMH}$. This atlas of conserved $\mathrm{VMH}$ neuron populations provides an unbiased starting point for the analysis of VMH circuitry and function. 


\section{INTRODUCTION}

The ventromedial hypothalamic nucleus $(\mathrm{VMH}$, which primarily contains glutamatergic neurons) plays important roles in a variety of metabolic responses and in the control of behaviors relevant to panic, reproduction, and aggression. The $\mathrm{VMH}$ contains several anatomic subdivisions, including the dorsomedial and central VMH $\left(\mathrm{VMH}_{\mathrm{DM}}\right.$ and $\mathrm{VMH}_{\mathrm{C}}$, respectively, which control autonomic outputs and behavioral responses to emergencies (Lindberg, Chen, and Li 2013; Vander Tuig, Knehans, and Romsos 1982)), and the ventrolateral $\mathrm{VMH}\left(\mathrm{VMH}_{\mathrm{VL}}\right.$; known for roles in sexual and social behaviors (Hashikawa et al. 2017; Krause and Ingraham 2017)). The predominantly GABAergic tuberal region of the hypothalamus lies anterolateral to the core of the $\mathrm{VMH}$.

Each VMH subdivision mediates a variety of outputs and thus presumably contains multiple functionally-distinct cell types. For example, activating adult Nr5a1expressing $\mathrm{VMH}$ neurons (which includes most cells in the $\mathrm{VMH}_{\mathrm{DM}}$ and $\mathrm{VMH}_{\mathrm{C}}$ ) promotes panic-related behaviors, augments hepatic glucose output to increase blood glucose, and elevates energy expenditure (Meek et al. 2016, 201; Flak et al. 2020; Kunwar et al. 2015). In contrast, activating the subset of $\mathrm{VMH}_{\mathrm{DM}}$ cells that expresses leptin receptor (Lepr, which encodes the receptor for the adipose-derived, energy balance-controlling hormone, leptin ( $\mathrm{H}$. Chen et al. 1996; Tartaglia et al. 1995)) promotes energy expenditure without altering these other parameters (Sabatini et al. 2021; Meek et al. 2013; 2016). Hence, each VMH subregion may contain multiple discrete neuron populations that mediate unique functions.

To date, most analyses of $\mathrm{VMH}$ function have utilized $\mathrm{Nr} 5 \mathrm{a} 1$ or candidate markers that do not necessarily align with functionally and/or transcriptionally unique 
VMH cell types (Bingham et al. 2006). Thus, to understand VMH-controlled responses, we must use unbiased methods to define discrete subpopulations of $\mathrm{VMH}$ neurons, along with markers that permit their selective manipulation. Single-cell approaches (such as single-nucleus RNA-sequencing (snRNA-seq)) can identify neuronal populations in an unbiased manner, and have previously suggested parceling schemes for neurons in many brain areas, including the VMH (D.-W. Kim et al. 2019; Campbell et al. 2017; Habib et al. 2017). Many such analyses define large numbers of highly interrelated cell populations of unclear functional significance and conservation, however (D.-W. Kim et al. 2019; R. Chen et al. 2017; Lam et al. 2017). Determining functions for dozens of cell populations that lie in the same anatomic region and which possess overlapping gene expression profiles (i.e., that don't contain unique marker genes) would represent a daunting task.

In the present study we use Translating Ribosome Affinity Purification with RNAsequencing (TRAP-seq) in mice together with snRNA-seq of mouse and macaque $\mathrm{VMH}$ neurons to define transcriptionally unique, anatomically discrete, conserved, and genetically tractable classes of $\mathrm{VMH}$ neurons. These include a distinct Lepr-expressing VMH neuron class, along with a set of glutamatergic $\mathrm{VMH}$ neurons that resides in the tuberal region. These findings define a starting point for the comprehensive analysis of VMH circuitry and function. 


\section{RESULTS}

\section{Combining snRNA-seq with Nr5a1-directed TRAP-seq defines mouse VMH neuron}

\section{populations}

To define neuronal populations within the mouse $\mathrm{VMH}$ in an unbiased manner, we microdissected the $\mathrm{VMH}$ of mice and subjected 10 individual tissue samples to snRNA-seq using the 10x Genomics platform (Fig. 1A), collecting a total of 42,040 nuclei that passed quality control (Fig. S1A-C). The recovered nuclei included all major CNS cell types (Fig. S1, see Methods for clustering and cell type identification details), including 21,585 neurons that comprised 37 distinct neuronal populations (Fig. 1B, C).

Many adult $\mathrm{VMH}$ neurons express $\mathrm{Nr} 5 \mathrm{a} 1$ (which encodes the transcription factor, SF1 (Cheung et al. 2013)) and/or Fezf1 (Kurrasch et al. 2007), whose detection was restricted to a confined cluster of neurons in UMAP space (Fig. 1D). Although essentially all $\mathrm{VMH}$ neurons express $\mathrm{Nr} 5 \mathrm{a} 1$ during development, only a subset of $\mathrm{VMH}$ cells express Nr5a1 and/or Fezf1 in adult animals (Cheung et al. 2013; Kurrasch et al. 2007). Furthermore, the inherent noise in snRNA-seq data risks false positives and negatives when using only one or two genes for cell-type identification. To ensure that we identified all VMH cell groups for our analysis, we performed TRAP-seq using Nr5a1-Cre;Rosa26 ${ }^{\text {GFP-L10a }}$ mice, in which the early developmental expression of Nr5a1Cre promotes the permanent expression of tagged ribosomes across the VMH (Figure 1E). TRAP-seq identified 4,492 transcripts significantly enriched in cells marked by the developmental expression of Nr5a1, including Nr5a1 and Fezf1 (Fig. 1F, Table 1). Applying this broader VMH-enriched transcriptome to our snRNA-seq clusters revealed 6 populations of neurons (clusters $6-11$; corresponding to the populations with highest 
Nr5a1 and Fezf1 expression) that contain VMH neurons. (Fig 1G,H). Importantly, Nr5a1-negative cells (clusters 6 and 7) were identified as $\mathrm{VMH}$ by their enrichment of Nr5a1-Cre TRAP-seq genes (Fig. 1H), suggesting they were developmentally labeled by $\mathrm{Nr} 5 \mathrm{a} 1-\mathrm{Cre}$; these cells presumably reside in the $\mathrm{VMH}_{\mathrm{VL}}$ (which expresses $\mathrm{Nr} 5 \mathrm{a} 1$ during development, but not in adulthood).

To compare TRAP-seq and snRNA-seq results, we performed "pseudo-TRAP" on pseudobulk samples of our snRNA-seq data, aggregated by cell type (i.e., VMH clusters vs. non-VMH clusters; see Table 2 for enrichment results). While many genes were enriched in both datasets $(1,977)$, more were specific to one method (Fig. 1l, 2,354 genes specific to TRAP and 2,828 specific to pseudo-TRAP). Notably, enrichment was largely a function of expression level: the genes that were enriched in both datasets were highly expressed in both, while the genes that were specific to TRAP or pseudoTRAP were highly expressed in their enriched dataset, but expressed at lower levels in the other (Fig. 1J). This suggests that the two methods may illuminate partially distinct aspects of the transcriptome-TRAP-seq for ribosome-associated genes and snRNAseq for nuclear-enriched and nascent transcripts—and that their combined use provides a more comprehensive view of cellular state. Finally, many of the genes enriched in both datasets were limited to one or a few populations, highlighting the heterogeneity of gene expression across VMH cell types, even for prominent VMH marker genes (Fig. $1 \mathrm{~K})$.

To understand the landscape of mouse $\mathrm{VMH}$ neuron populations in more detail, we subjected the VMH neurons in clusters 6-11 (Fig $1 \mathrm{H})$ to further clustering, which identified 24 transcriptionally-defined neuronal populations (Fig 2A-C). The cell groups 
that we identified are largely consistent with a recently published $\mathrm{VMH}_{\mathrm{VL}}$-focused singlecell (sc) RNA-seq study (Figure S3) (D.-W. Kim et al. 2019). While our study identified 24 clusters whose major markers were evenly distributed between the dorsomedial and ventrolateral compartments of the $\mathrm{VMH}$, Kim et al. identified 31 clusters with a bias toward populations with $\mathrm{VMH}_{\mathrm{VL}}$ markers. Integrating the datasets (Fig. S3) revealed their broad correspondence, with highly correlated expression profiles (Fig. S3B) and shared marker genes (Fig. S3D). The omission of Nfib-marked populations from the Kim et al. analysis (D.-W. Kim et al. 2019) represented a notable difference between our analyses, however. In our reanalysis of their data, we found that Nfib-marked cells were present in their samples, but were filtered out before the final VMH clustering (Fig. S3). A previous scRNA-seq study of the neighboring ARC also mapped a neuron population marked by Nfib expression to the $\mathrm{VMH}$, however (Campbell et al. 2017), and these cells correlated to our Nfib-marked VMH neuron populations (Fig. S4).

\section{A simplified parceling scheme defines anatomically-distinct VMH neuron}

\section{populations}

Hierarchical clustering and marker gene analysis for our 24 mouse VMH neuron clusters using CELLEX (Timshel, Thompson, and Pers 2020) revealed that many cell groups were highly related to other $\mathrm{VMH}$ neuron clusters (Fig. 2D, E; Table 3).

Furthermore, many of these populations share marker gene expression to an extent that renders it impossible to specifically manipulate single populations given current approaches that use a single gene for cell type manipulation (e.g., Cre-based mouse models) (Fig. 2D, E). To identify classes of genetically distinguishable cells, we cut the 
hierarchical tree at different levels and measured the maximum pairwise expression correlation to highlight the level at which few pairs of clusters exhibited highly correlated transcriptomes. We found that 6 classes represented the largest number of classes that retained minimal correlated expression (Fig. 2F). To avoid confusion, we refer to these as $\mathrm{VMH}$ neuron classes, while referring to the cell groups of which each class is composed as clusters or subpopulations. The mean silhouette width (a measure of clustering robustness) for the various tree cuts also supported the use of 6 classes (Fig. S2), and these 6 classes corresponded to the cluster designations from the broader neuron dataset (Fig. 2G). Hence, a parceling scheme for mouse VMH neurons that contains 6 classes, each composed of highly similar subpopulations, captures the transcriptional patterns of the $\mathrm{VMH}$. Importantly, this approach identified numerous specific marker genes (e.g., DIk1, Esr1, Nfib, Foxp2, Fezf1, and Lepr) for each class of mouse VMH neurons (Fig. 2D, H, Table 4), which should facilitate their manipulation and study.

Consistent with the distinct nature of these $6 \mathrm{VMH}$ neuron classes and the utility of this parceling scheme, each class demonstrated a circumscribed anatomic distribution. As previously reported (Lee et al. 2014; Persson-Augner et al. 2014), Dlk1and Esr1-expressing neurons $\left(\mathrm{VMH}^{\mathrm{DIk} 1}\right.$ and $\mathrm{VMH}^{\mathrm{Esr} 1}$, respectively) map to the $\mathrm{VMH}_{\mathrm{VL}}$. In contrast, Lepr-expressing cells lie within the core of the $\mathrm{VMH}_{\mathrm{DM}}$ (Elmquist et al. 1998) and the expression of marker genes for the Nfib-marked clusters $\left(\mathrm{VMH}^{\mathrm{Nrib}}\right)$ resides in the most dorsomedial compartment of the $\mathrm{VMH}_{\mathrm{DM}}$ (Fig. S5). Fezf1-marked populations $\left(\mathrm{VMH}^{\mathrm{Fezf1}}\right)$ include cells with similarity to both ventrolateral $\mathrm{VMH}^{\mathrm{Dk} 1}$ and dorsomedial $\mathrm{VMH}^{\mathrm{Lepr}}$ neurons, and many $\mathrm{VMH}^{\mathrm{Fezf1}}$ neurons lie in $\mathrm{VMH}_{\mathrm{C}}$, in the transition between the 
dorsomedial and ventrolateral zones of the $\mathrm{VMH}$. Markers for the Foxp2-expressing populations $\left(\mathrm{VMH}^{\mathrm{Foxp2}}\right)$ reside anterior and lateral to the core of the $\mathrm{VMH}$, in the socalled tuberal region (Fig. S6). Thus, the major VMH classes identified by our analysis each map to specific and distinct anatomic locations, consistent with their unique genetic signatures.

\section{Lepr-directed TRAP-seq analysis of VMH ${ }^{\text {Lepr }}$ cells}

Given that Lepr-expressing $\mathrm{VMH}$ neurons mediate only a subset of $\mathrm{VMH}_{\mathrm{DM}}$ functions, we were intrigued by the finding that unbiased snRNA-seq identified a distinct class of $\mathrm{VMH}_{\mathrm{DM}}$ cells marked by Lepr $\left(\mathrm{VMH}^{\mathrm{Lepr}}\right.$ neurons), consistent with a specialized role for Lepr-expressing VMH cells (Minokoshi, Haque, and Shimazu 1999; Toda et al. 2013; Noble et al. 2014; Gavini, Jones, and Novak 2016). The finding that Leprexpressing $\mathrm{VMH}$ neurons map onto a single $\mathrm{VMH}$ neuron class also suggests a uniformity of function for these cells, which contrasts with the situation in other brain regions (such as the neighboring $A R C$, where multiple cell types with opposing functions (e.g., Agrp and Pomc cells) express Lepr (Campbell et al. 2017)). We utilized TRAP-seq to assess the extent to which Lepr-expressing VMH neurons correspond to the $\mathrm{VMH}^{\text {Lepr }}$ clusters and to compare the genetic program of Lepr-expressing $\mathrm{VMH}$ neurons with gene expression in snRNA-seq-defined $\mathrm{VMH}$ neuron classes and subpopulations.

TRAP-seq analysis of microdissected VMH tissue from Lepr $^{\text {Cre }} ;$ Rosa26 ${ }^{\text {eGFP-L10a }}$ animals (Leshan et al. 2006) resulted in the enrichment of transcripts from Lepr Cre neurons that lie in VMH-adjacent brain areas, including Agrp-, Ghrh-, Pomc- and Ntsexpressing cells from the ARC and lateral hypothalamic area (LHA) (Fig. S7). To more 
closely restrict our TRAP-seq analysis to Lepr-expressing neurons that reside in the VMH, we used a recently developed mouse line that expresses eGFP-L10a only in cells that contain both Cre and Flp recombinases (Sabatini et al. 2021). Because VMH neurons contain vGLUT2 (encoded by Slc17a6), while most Lepr-expressing neurons in the neighboring $A R C$, dorsomedial hypothalamus $(\mathrm{DMH})$, and LHA do not (Vong et al. 2011), we crossed a Slc17a6 $6^{\text {Flpo }}$ mouse line to $L e p r^{\text {Cre }}$ and $R C F L^{\text {eGFP-L10 }}$ to produce Slc17a6 ${ }^{\text {Flpo }}$; Lepr ${ }^{\text {Cre. }} ; R C F L^{\text {eGFP-L10 }}$ (Lepr $^{\text {Slc17a6 }}$-L10a) mice. In these mice, mediobasal hypothalamic eGFP-L10a was largely restricted to the VMH (Fig. 3A) (Sabatini et al. 2021).

We microdissected the VMH of Lepr ${ }^{\text {Slc17a6 }}$-L10a mice and performed TRAP-seq, identifying 3,580 transcripts that were enriched in the TRAP material relative to the supernatant (Fig. 3C, Table 5). Importantly, Lepr itself was not enriched, suggesting that we successfully purified ribosome-associated mRNA from Lepr-expressing VMH cells away from that derived from other Lepr-expressing populations (Fig. 3B). We found that most non-VMH genes enriched in our conventional ( $\mathrm{Lepr}^{\mathrm{Cre}}$-only) TRAP-Seq (including Agrp, Nts, and Ghrh) were not enriched in this analysis (Fig S7). Pomc and Prlh remained somewhat enriched, however (Fig. S7), suggesting that Lepr-expressing DMH Prlh cells are glutamatergic (Dodd et al. 2014), and consistent with the finding that some ARC Pomc cells express Slc17a6 (Jones et al. 2019).

As expected, we observed a high degree of concordance between snRNA-seqdefined gene expression in neurons of the $\mathrm{VMH}^{\mathrm{Lepr}}$ cluster with gene expression in Lepr-expressing VMH neurons by TRAP-seq. We identified 3,576 genes enriched in VMH-centered Lepr ${ }^{\text {Slc17a6 }}$ TRAP-seq material (Fig. 3C), 1,174 of which were also 
enriched in pseudo-TRAP analysis of the neurons assigned to the $\mathrm{VMH}^{\mathrm{Lepr}}$ population (Table 6). Among the top enriched genes shared by these two methods were Gpr149, Rai14, and Tnfrsf8 (Fig. 3F), which exhibit a similar $\mathrm{VMH}_{\mathrm{DM}}$-centered expression pattern as Lepr (Fig. 3G-I). As with the Nr5a1-Cre TRAP-seq, enrichment was largely a function of expression level: the genes that were enriched in both datasets were highly expressed in both, while the genes that were specific to TRAP or pseudo-TRAP were highly expressed in their enriched dataset, but more lowly expressed in the other.

Notably, gene ontology analysis of the common genes revealed many terms related to synaptic function, while genes unique to TRAP-seq were enriched for ribosomal and mitochondrial function (data not shown).

We mapped enriched genes from Lepr ${ }^{\mathrm{Slc17a6}}$ TRAP-seq to the gene expression profiles of our snRNA-seq-defined $\mathrm{VMH}$ neuron populations (Fig 3F), revealing the bias of Lepr ${ }^{\text {Slc17a6 }}$ TRAP-seq gene expression toward $\mathrm{VMH}^{\text {Lepr }}$ neurons and the exclusion of markers from other VMH ${ }^{\text {Lepr }}$ cells from Lepr ${ }^{\text {Slc17a6 }}$ TRAP-seq-enriched genes. Thus, this analysis demonstrates that Lepr-expressing $\mathrm{VMH}$ neurons map specifically to $\mathrm{VMH}^{\text {Lepr }}$ cell clusters, suggesting that they represent a transcriptionally and functionally unique set of neurons.

\section{Conservation of VMH neuronal populations across species}

While many previous studies of $\mathrm{VMH}$ neuron function have suggested that this brain region contains neurons that could represent therapeutic targets to aid people with obesity, diabetes, and other diseases, most of these studies have been performed in mice (Hashikawa et al. 2017; Flak et al. 2020; Meek et al. 2013; K. W. Kim et al. 2012). We know little about the cross-species conservation of VMH cell populations, however. 
To assess the potential conservation of $\mathrm{VMH}$ neuron populations across species, we microdissected macaque (Macaca mulatta) VMH and performed snRNA-seq using the same techniques as for mouse $\mathrm{VMH}$ (Fig. S8). A subset of macaque neurons expressed NR5A1 and/or FEZF1 and exhibited similar gene expression profiles to our mouse Nr5a1-Cre TRAP-seq enriched genes (Fig. S9), suggesting that these cells represent the macaque $\mathrm{VMH}$ and indicating that the mouse and macaque $\mathrm{VMH}$ share similar global gene expression signatures.

Graph-based clustering of the macaque $\mathrm{VMH}$ neurons yielded 7 populations with unique marker genes (with the partial exception of two related ESR1-expressing cell types) (Figure 4B-E). Most of these macaque populations, including populations marked by LEPR, FOXP2, NFIB and ESR1 $\left(\mathrm{VMH}^{\mathrm{LEPR}}, \mathrm{VMH}^{\mathrm{FOXP} 2}, \mathrm{VMH}^{\mathrm{NFIB}}\right.$, and $\mathrm{VMH}^{\mathrm{ESR} 1}$, respectively), have presumptive orthologs in the mouse (Fig. 4D, see Table 7 for a complete list of markers). While populations marked by DLK1 and FEZF1 were absent from this analysis, the macaque $\mathrm{VMH}$ contained a population marked by $Q R F P R$ expression $\left(\mathrm{VMH}^{\mathrm{QRFPR}}\right)$.

To determine whether the macaque populations were orthologous to those from the mouse, we first compared expression of orthologous genes among mouse and macaque cell clusters. We found that all macaque populations had clear correlates in the mouse, which mapped according to their marker genes (as expected) (Fig. 4F, S10D). Notably, the macaque $\mathrm{VMH}^{\mathrm{QRFPR}}$ population correlated with clusters from both $\mathrm{VMH}^{\mathrm{DIk} 1}$ and $\mathrm{VMH}^{\mathrm{Fezf1}}$ classes in the mouse, suggesting that $\mathrm{VMH} \mathrm{HRFPR}^{\mathrm{QRF}}$ contains orthologs of the mouse $\mathrm{VMH}^{\mathrm{DIk} 1}$ and $\mathrm{VMH}^{\mathrm{Fezf} 1}$ classes. Projecting the mouse or macaque cluster labels onto the other species using Seurat anchors (see Methods for 
more detail) yielded similar results (Fig. S10A). All major classes from mouse and macaque projected with high confidence onto the equivalent major classes of the other species, and the macaque $V M H^{Q R F P R}$ population was represented by both mouse $\mathrm{VMH}^{\mathrm{Dlk} 1}$ and $\mathrm{VMH}^{\mathrm{Fezf1}}$ cells (Fig. S10A).

To generate an atlas of conserved mouse and macaque VMH populations, we integrated the mouse and macaque data and clustered the merged dataset using the Seurat CCA framework (Fig. 5A-B, see Methods for more detail). This analysis revealed populations of $\mathrm{VMH}$ neurons that each contained mouse and macaque cells in roughly equal proportions (Fig. 5E). As predicted, neurons from the macaque VMH ${ }^{\mathrm{LEPR}}$, $\mathrm{VMH}^{\mathrm{FOXP2}}, \mathrm{VMH}^{\mathrm{NFIB}}$, and $\mathrm{VMH}^{\mathrm{ESR} 1}$ classes mapped directly with murine $\mathrm{VMH}^{\mathrm{Lepr}}$, $\mathrm{VMH}^{\mathrm{Foxp} 2}, \mathrm{VMH}^{\mathrm{Nfib}}$ and $\mathrm{VMH}^{\mathrm{Esr} 1}$ classes, respectively (Fig. 5G). We examined the potential co-expression of conserved marker genes for $\mathrm{VMH}^{\mathrm{LEPR}}$ cells by in situ hybridization (ISH) for ACVR1C in the macaque hypothalamus (Fig. S11), confirming ACVR1C is co-expressed with $L E P R$ in the macaque $\mathrm{VMH}_{\mathrm{DM}}$. We also examined the potential co-expression of SLC17A8 (which marks $\mathrm{VMH}^{\mathrm{NFIB}}$ ) with LEPR. SLC17A8 identified a population of cells that lay at the medial extreme of the macaque $\mathrm{VMH}_{\mathrm{DM}}$, corresponding to the most dorsomedial aspect of the rodent $\mathrm{VMH}_{\mathrm{DM}}$, as for murine $\mathrm{VMH}^{\mathrm{Nfib}}$ cells; these cells did not colocalize with LEPR-expressing cells (Fig. S11).

This analysis also identified macaque populations that mapped with the murine $\mathrm{VMH}^{\mathrm{Dlk} 1}$ and $\mathrm{VMH}^{\mathrm{Fezf} 1}$ populations; these derived mainly from the macaque $\mathrm{VMH}^{\mathrm{QRFPR}}$ population (Fig. 5G). Notably, FEZF1 and DLK1 were poorly enriched in the macaque $\mathrm{VMH}^{\mathrm{QRFPR}}$ population (Fig. 5D), while mouse Qrfpr expression was biased toward the $\mathrm{VMH}^{\mathrm{Esr}}$ populations (Fig. 5D). Despite differences in some marker genes, however, the 
orthologous macaque and mouse VMH neuron populations share dozens of other genes across species (Fig. 5H, Table 8, Fig. S10B), suggesting that the mouse and macaque cells in each group represent similar cell types. GO analysis of common marker genes (Table 9) revealed that most of these mediate core neuronal functions, such as ion channel activity (Fig. S10C). Thus, while some marker genes vary across species, the mouse $\mathrm{VMH}$ populations have close orthologs in the macaque $\mathrm{VMH}$ based upon their gene expression profiles. 


\section{DISCUSSION}

We combined TRAP- and snRNA-seq analysis of the VMH to identify 24 mouse neuronal populations with complex interrelations. These 24 populations represented six distinct cell classes that demonstrated unique anatomic distribution patterns. The main $\mathrm{VMH}$ classes were highly conserved between the mouse and the macaque in terms of gene expression profiles and anatomic distribution within the $\mathrm{VMH}$. This atlas of conserved $\mathrm{VMH}$ neuron populations provides an unbiased and tractable starting point for the analysis of $\mathrm{VMH}$ circuitry and function.

Having many populations of $\mathrm{VMH}$ neurons with highly related gene expression profiles complicates the functional analysis of $\mathrm{VMH}$ cell types, suggesting the importance of simplifying the map of these heterogeneous populations to permit their manipulation and study. By using hierarchical clustering, we were able to identify six maximally unrelated classes of $\mathrm{VMH}$ neurons with distinct gene expression signatures. These six discrete transcriptionally-defined $\mathrm{VMH}$ neuronal classes demonstrated distinct anatomic distribution patterns (three located in the $\mathrm{VMH}_{\mathrm{DM}}$, two in the $\mathrm{VMH}_{\mathrm{VL}}$, and one in the tuberal region), revealing a transcriptional basis for the previously-suggested functional architecture of the $\mathrm{VMH}$.

Our VMH cell populations were similar to those previously described by Kim et al., although they more finely split the $\mathrm{VMH}_{\mathrm{VL}}$ populations than did we (presumably because their dissection bias toward the $\mathrm{VMH}_{\mathrm{VL}}$ yielded more $\mathrm{VMH}_{\mathrm{VL}}$ neurons) (D.-W. Kim et al. 2019). We were able to more clearly distinguish among $\mathrm{VMH}_{\mathrm{DM}}$ cell types in our $\mathrm{VMH}_{\mathrm{DM}}$-focused analysis, however, including by dividing the $\mathrm{VMH}_{\mathrm{DM}}$ neurons that they identified into two major classes $\left(\mathrm{VMH}^{\mathrm{Lepr}}\right.$ and $\left.\mathrm{VMH}^{\mathrm{Fezf1}}\right)$, as well as identifying a third 
$\mathrm{VMH}_{\mathrm{DM}}$ population $\left(\mathrm{VMH}^{\mathrm{Nfib}}\right)$ absent from their analysis. Interestingly, the $\mathrm{VMH}^{\mathrm{Nfib}}$ population was quite distinct from the more closely related $\mathrm{VMH}^{\text {Lepr }}$ and $\mathrm{VMH}^{\text {Fezf1 }}$ $\mathrm{VMH}_{\mathrm{DM}}$ cell types, suggesting a potentially divergent function for this most dorsomedial of the $\mathrm{VMH}_{\mathrm{DM}}$ populations.

Our mouse VMH cell types mapped clearly onto specific populations of macaque VMH neurons, revealing the utility of the mouse as a surrogate for the primate in terms of $\mathrm{VMH}$ cell types and, presumably, function. While the macaque single $\mathrm{VMH}^{\mathrm{QRFRP}}$ population mapped to two mouse classes $\left(\mathrm{VMH}^{\mathrm{Fezf} 1}\right.$ and $\left.\mathrm{VMH}^{\mathrm{Dlk} 1}\right)$ by orthologous gene expression, these mouse classes are closely related to each other (transcriptionally and anatomically) and the macaque cells from VMH ${ }^{\text {QRFRP }}$ segregate to distinct cell clusters defined by Dlk1 and Fezf1 in the mouse when we integrated and reclustered the mouse and macaque cell data. Also, while $D L K 1$ is not specific to the macaque $\mathrm{VMH}^{\mathrm{QRFPR}}$ cells, the otherwise similar gene expression profiles of mouse and macaque cells that map to $\mathrm{VMH}^{\mathrm{Dlk1}}$ or $\mathrm{VMH}^{\mathrm{Fezf} 1}$ populations suggests the conserved nature of these cell types across species.

While not all Lepr-expressing cell types track in the brain across species (e.g., preproglucagon $(\mathrm{Gcg})$-containing NTS neurons in the mouse express Lepr, rat NTS Gcg cells do not (Huo et al. 2008)), mouse VMH ${ }^{\text {Lepr }}$ neurons map directly with macaque $\mathrm{VMH}^{\mathrm{LEPR}}$ neurons by all of the measures that we examined. The finding that Lepr/LEPR expression marks a unique and conserved cell type in rodent and primate is consistent with the notion that this class of $\mathrm{VMH}$ neuron mediates a discrete component of $\mathrm{VMH}$ function, as suggested by previous work in the mouse demonstrating roles for Lepr- 
expressing $\mathrm{VMH}$ neurons in the control of energy balance, but not glucose production or panic-like behaviors (Meek et al. 2013; 2016; Sabatini et al. 2021).

While the tuberal region contains more GABAergic than glutamatergic neurons, this region projects to similar target areas as does the core $\mathrm{VMH}$ and contains substantial numbers of neurons marked by Nr5a1-Cre activity. Hence, although $\mathrm{VMH}^{\mathrm{Foxp} 2}$ cells lie in the tuberal region, their glutamatergic nature, their marking by Nr5a1-Cre activity, and the finding that they are transcriptional most similar to other $\mathrm{VMH}$ populations mark $\mathrm{VMH}^{\mathrm{Foxp} 2}$ cells as $\mathrm{VMH}$ neurons. While few data exist to suggest the physiologic roles played by these cells, it will be interesting to manipulate $\mathrm{VMH}^{\text {Foxp2 }}$ neurons to determine their function.

The identification of distinct transcriptionally-defined VMH cell populations provides the opportunity to develop new tools that can be used to understand the nature and function of $\mathrm{VMH}_{\mathrm{DM}}$ cell types and their roles in metabolic control. The finding that the major VMH cell classes found in the mouse are present in the macaque support the use of the mouse to study the metabolic functions of the $\mathrm{VMH}$ as a means to identify potential therapeutic targets for human disease. It will be important to use these findings to dissect functions for subtypes of $\mathrm{VMH}$ cells, which may represent targets for the therapy of diseases including obesity and diabetes. 


\section{Methods}

Animals

Mice were bred in the Unit for Laboratory Animal Medicine at the University of Michigan. These mice and the procedures performed were approved by the University of Michigan Committee on the Use and Care of Animals and in accordance with Association for the Assessment and Approval of Laboratory Animal Care (AAALAC) and National Institutes of Health $(\mathrm{NIH})$ guidelines. Mice were provided with ad libitum access to food (Purina Lab Diet 5001) and water in temperature-controlled $\left(25^{\circ} \mathrm{C}\right)$ rooms on a $12 \mathrm{~h}$ light-dark cycle with daily health status checks.

Nr5a1-Cre (Jax: 012462) (Dhillon et al. 2006) and Foxp2 ${ }^{\text {IRES-Cre }}$ (Jax: 030541) (Rousso et al. 2016) mice were obtained from Jackson Laboratories. Rosa26 ${ }^{\text {CAG-LSL- }}$ eGFP-L10a, Lepr ${ }^{\text {Cre }}$ (Jax: 032457), SIC17a6 $6^{F 1 p o}$ and RCFL ${ }^{\text {EGFP-L10a }}$ mice have been described previously(Leshan et al. 2006; Krashes et al. 2014; Sabatini et al. 2021).

Nonhuman primate tissue was obtained from the Tissue Distribution Program at ONPRC. Animal care is in accordance with the recommendations described in the Guide for the Care and Use of Laboratory Animals of the NIH and animal facilities at the Oregon National Primate Research Center (ONPRC) are accredited by AAALAC.

Tissue prep, cDNA amplification and library construction for 10x snRNA-seq

Mice were euthanized using isoflurane and decapitated, the brain was subsequently removed from the skull and sectioned into $1 \mathrm{~mm}$ thick coronal slices using a brain matrix. The VMH was dissected out and flash frozen in liquid $\mathrm{N}_{2}$. Nuclei were isolated as previously described (Habib et al. 2017) with modifications as follows. On 
the day of the experiment, frozen VMH (from $2-3$ mice) was homogenized in Lysis Buffer (EZ Prep Nuclei Kit, Sigma) with Protector RNAase Inhibitor (Sigma) and filtered through a $30 \mu \mathrm{m}$ MACS strainer (Myltenti). Strained samples were centrifuged at $500 \mathrm{rcf}$ x 5 minutes and pelleted nuclei were resuspended in washed with wash buffer (10mM Tris Buffer $\mathrm{pH} 8.0,5 \mathrm{mM} \mathrm{KCl}, 12.5 \mathrm{mM} \mathrm{MgCl}_{2}, 1 \% \mathrm{BSA}$ with RNAse inhibitor). Nuclei were strained again and recentrifuged at 500rcf $\times 5$ minutes. Washed nuclei were resuspended in wash buffer with propidium iodide (Sigma) and stained nuclei underwent FACS sorting on a MoFlo Astrios Cell Sorter. Sorted nuclei were centrifuged at 100rcf $\times 6$ minutes and resuspended in wash buffer to obtain a concentration of $750-$ 1200 nuclei/uL. RT mix was added to target $\sim 10,000$ nuclei recovered and loaded onto the 10x Chromium Controller chip. The Chromium Single Cell 3' Library and Gel Bead Kit v3, Chromium Chip B Single Cell kit and Chromium i7 Multiplex Kit were used for subsequent RT, cDNA amplification and library preparation as instructed by the manufacturer. Libraries were sequenced on an Illumina HiSeq 4000 or NovaSeq 6000 (pair-ended with read lengths of $150 \mathrm{nt}$ ) to a depth of at least 50,000 reads/cell.

\section{snRNA-seq data analysis}

Count tables were generated from the FASTQ files using cellranger and analyzed in R 3.6 .3 using the Seurat 3 framework. Genes expressed in at least 4 cells in each sample and were not gene models (starting with "Gm") or located on the mitochondrial genome were retained. Cells with at least 500 detected genes were retained. Doublets were detected using Scrublet (Wolock, Lopez, and Klein 2019). For each 10x run, the expected number of doublets was predicted using a linear model 
given 10x data of the detected doublet rate and the number of cells. Then, each cell was given a doublet score with Scrublet and the $\mathrm{n}$ cells (corresponding to the expected number of doublets) with the top scores were removed.

The data was then normalized using scran (Lun, McCarthy, and Marioni 2016) and centered and scaled for each dataset independently and genes that were called variable by both Seurat FindVariableFeatures and sctransform (Hafemeister and Satija 2019) were input to PCA. The top PCs were retained at the "elbow" of the scree plot (normally $15-30$, depending on the dataset) and then used for dimension reduction using UMAP and clustering using the Seurat FindNeighbors and FindClusters functions. Both were optimized for maximizing cluster consistency by clustering over a variety of conditions: first, varying the number of neighbors from 10 to the square root of the number of cells while holding the resolution parameter in FindClusters at 1 and finding the clustering that maximized the mean silhouette score; then, this number of neighbors was held fixed while varying the resolution parameter in FindClusters from 0.2 upward in steps of 0.2 until a maximal mean silhouette score was found. Clusters were then hierarchically ordered based on their Euclidean distance in PC space and ordered based on their position in the tree.

Marker genes were found using the Seurat function FindAllMarkers for each sample with resulting $p$-values combined using the logitp function from the metap package or using CELLEX 1.0.0. Cluster names were chosen based on genes found in this unbiased marker gene search and known marker genes.

From the all-cell data, cell types were predicted using gene set enrichment analysis from the marker genes and a manually curated set of genes known to mark 
specific CNS cell types. From this, clusters that were highly enriched for markers from 2 (or more) distinct cell types were labeled as "doublets" and those with no enrichment were labeled as "junk", the remaining clusters were labeled based on their lone CNS cell type.

To predict $\mathrm{VMH}$ neurons from all neurons, we first found genes significantly enriched in the bead fraction in Nr5a1-Cre TRAP-seq (see below for details) and expressed above 1 count per million. The scaled count matrix containing these genes was then used as input to PCA. The magnitude of the first principal component (loading) was then used to generate a VMH similarity score and the clusters that had a high Nr5a1-Cre TRAP loading, were glutamatergic (express Slc17a6 and not Gad1 or Slc32a1 above the mean value), and expressed either Nr5a1 or Fezf1 above the mean value were included as presumptive $\mathrm{VMH}$.

\section{TRAP-seq analysis}

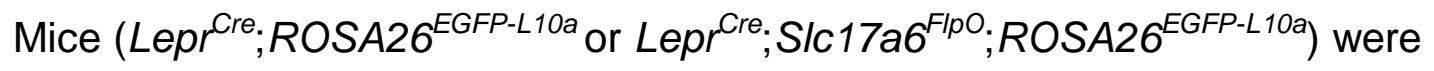
euthanized and decapitated, the brain was subsequently removed from the skull and sectioned into $1 \mathrm{~mm}$ thick coronal slices using a brain matrix. The $\mathrm{VMH}$ or hypothalamus was dissected and homogenized in lysis buffer. Between 15-20 mice were used for TRAP experiments. GFP-tagged ribosomes were immunoprecipitated and RNA isolated as previously described (Allison et al. 2018). RNA was subject to ribodepletion and the resultant mRNA was fragmented and copied into first strand cDNA. The products were purified and enriched by PCR to create the final cDNA library. Samples were sequenced 
on a 50-cycle single end run on a HiSeq 2500 (Illumina) according to manufacturer's protocols.

FASTQ files were filtered using fastq_quality_filter from fastx_toolkit to remove reads with a phred score $<20$. Then reads were mapped using STAR with a custom genome containing the Ensembl reference and sequences and annotation for Cre and EGFP:L10a (and Flpo in the RCFL::eGFP-L10a dataset). Count tables were generated using the STAR --quantMode GeneCounts flag.

Count tables were analyzed in R 3.6.3 and were subject to quality control to ensure read adequate library size (20-30 million reads), enrichment of positive control genes (e.g. EGFP:L10a and/or Cre, Nr5a1), and appropriate sample similarity in both hierarchical clustering of Euclidean distance and TSNE/UMAP space (e.g. bead samples are more similar to one another than to any sup sample). All samples passed quality control. Enriched genes were determined using DESeq2 including an effect of sample pair in the model to account for pairing of the bead-sup samples ( pair + cells).

$\underline{\text { Integration with published data }}$

Count tables from Kim et al. (D.-W. Kim et al. 2019) were downloaded from https://data.mendeley.com/datasets/ypx3sw2f7c/3 and count tables from Campbell et al. (Campbell et al. 2017) were downloaded from

\section{https://www.ncbi.nlm.nih.gov/geo/query/acc.cgi?acc=GSE93374. Note: only the 10x}

data from Kim et al. was used. The data was then preprocessed and clustered in the same way as above, though some samples were removed from the Kim et al. dataset for low mean read depth that confounded clustering (Samples 2018_0802, 2018_0803, 
2018_0812_1, 2018_0812_2, and 2018_0812_3). For the Kim et al. dataset, cells were clustered as above (FindNeighbors then FindClusters), and neuron clusters were predicted using WGCNA to identify correlated gene expression modules. The modules contained dozens of genes that mapped clearly onto a small set of clusters in UMAP space and—based on known marker genes—corresponded to the most prevalent cell types in the brain (e.g. neurons, astrocytes, microglia, oligodendrocytes, etc.). For the Campbell et al. data, neurons were labeled in the metadata from the authors, so neuronal barcodes were simply selected based on their annotation. For both neuronal datasets, $\mathrm{VMH}$ neurons were predicted using the same procedure as above: clusters that were glutamatergic, Fezf1/Nr5a1-expressing, and similar to Nr5a1-Cre TRAP-seq. This corroborated the clusters called VMH in both datasets by the original authors, with the exception of the Nfib populations in the Kim et al. dataset that was not called VMH and therefore not assigned a cluster name; we refer to these cells as (Missing) in our integrated dataset.

To find shared populations across datasets we took 2 approaches. First, variable genes were found for both datasets using the Seurat FindVariableFeatures function. Then, the pair-wise Pearson correlation was found for the mean scaled expression in each cluster in each dataset for the set of genes called variable in both datasets. Additionally, we used the Seurat FindTransferAnchors and IntegrateData functions to generate a merged dataset that was then subjected to PCA, UMAP reduction, and clustering in the same way as above. These new clusters containing cells from both datasets were then manually named using marker genes from the original datasets (e.g. Dlk1, Esr1, Satb2, Lepr, Nfib, Foxp2, etc.). 


\section{$\underline{\text { Immunostaining }}$}

Animals were perfused with phosphate buffered saline for five minutes followed by an additional five minutes of $10 \%$ formalin. Brains were then removed and post-fixed in $10 \%$ formalin for 24 hours at room temperature, before being moved to $30 \%$ sucrose for 24 hours for a minimum of 24 hours at room temperature. Brains were then sectioned as $30 \mu \mathrm{m}$ thick free-floating sections and stained. Sections were treated with blocking solution (PBS with $0.1 \%$ triton, 3\% normal donkey serum; Fisher Scientific) for at least 1 hour. The sections were incubated overnight at room temperature in was preformed using standard procedures. The following day, sections were washed and incubated with fluorescent secondary antibodies with species-specific Alexa Fluor-488 or -568 (Invitrogen, 1:250) to visualize proteins. Primary antibodies used include: GFP (1:1000, \#1020, Aves Laboratories) and NFIA (1:500, \#PA5-35936, Invitrogen). Images were collected on an Olympus BX51 microscope. Images were background subtracted and enhanced by shrinking the range of brightness and contrast in ImageJ.

\section{Macaque snRNA-seq}

Whole Rhesus macaque brains were obtained from the Tissue Distribution Program at ONPRC. Brains were centered within a chilled brain matrix (ASI Instruments, catalog \# MBM-2000C) and 2mm slices were obtained from rostral to caudal. Slices containing the hypothalamus were placed in saline and the $\mathrm{PVH}, \mathrm{ARC}, \mathrm{VMH}$ and $\mathrm{DMH}$ were punched out and samples were placed in pre-chilled tubes on dry-ice. Samples 
were stored at $-80 \mathrm{C}$ until shipment on dry ice. Nuclei were isolated from frozen macaque tissue as described above for mice.

The FASTQ files were mapped to the macaque genome (Mmul_10) using cellranger and count matrix files were analyzed in $\mathrm{R}$ using Seurat 3 as above, with the exception that gene models and genes mapping to the macaque mitochondrial genome were not removed. Macaque neurons were predicted using orthologs of mouse cell type marker genes and macaque $\mathrm{VMH}$ neurons were identified using macaque orthologs of Nr5a1-Cre TRAP-seq enriched genes. Orthologs were identified using Ensembl and only 1:1 orthologs were retained.

\section{Species integration}

The mouse and macaque datasets were integrated in a similar way to integrating the Kim et al. and Campbell et al. mouse datasets. First, the macaque genes were renamed to their mouse orthologs and only $1: 1$ orthologs and genes expressed in both species were retained. Importantly, because our dataset was biased toward the dorsomedial $\mathrm{VMH}$ and the Kim et al. dataset was biased toward the ventrolateral $\mathrm{VMH}$, we also included 4 randomly chosen samples from the Kim et al. data to get a more representative picture of shared VMH populations across species. The data was then preprocessed, normalized, and scaled in the same way as previously. The mouse and macaque data was then integrated using the Seurat FindTransferAnchors and IntegrateData functions and marker genes were found that were common across species by running FindAIIMarkers for each species separately and then using the metap logitp function to find genes that are significantly enriched. 


\section{Macaque in situ hybridization}

Whole Rhesus macaque brains were obtained from the Tissue Distribution Program at

ONPRC. Hypothalamic blocks fixed with $4 \%$ paraformaldehyde were incubated in glycerol prior to freezing with isopentane. Tissue was sectioned at $25 \mu \mathrm{m}$ using a freezing stage sliding microtome and free-floating sections were stored in glycerol cryoprotectant at $-20^{\circ} \mathrm{C}$. Tissue was mounted on slides prior to in situ hybridization, which was performed using ACD Bio RNAScope reagents (Multiplex Fluorescent Detection Kit v2, 323100) for Acvr1c (ACD 591481), Slc17a8 (ACD 543821-c2) and Lepr (ACD 406371-C3). Negative and positive control probes were included in all runs. Slides were imaged on an Olympus VS110 Slidescanner and processed using Visiopharm software.

Statistical Analysis

All data is displayed as mean +/- SEM. All plotting and statistical analysis was performed using $R$ 3.6.3. Specific statistical tests are listed in the figure legends.

\section{$\underline{\text { Resource availability }}$}

All mouse strains will be made available upon reasonable request.

\section{Data availability}

Sequencing data, count matrices, and metadata for all experiments are available through GEO at accession number GSE172207. This is currently private until acceptance for publication. 
Code availability

All analysis code will be available at github.com/alanrupp/affinati-2021.

Author contributions: AHA, PVS, AJT, CT, MK, SRL, and ACR researched and analyzed data. AHA, PVS, CL, SRL, DPO, PK, MGM and ACR designed experiments and wrote and edited the manuscript. All authors reviewed and edited the manuscript. MGM is the guarantor of the manuscript.

Acknowledgments: We thank Randy Seeley, Lotte Bjerre Knudsen, Kevin Grove, Mads Tang-Christensen, Christine Bjørn Jensen and members of the Myers and Olson labs for helpful discussions. Research support was provided by the Michigan Diabetes Research Center (NIH P30 DK020572, including the Molecular Genetics, Microscopy, and Animal Studies Cores), the Marilyn H. Vincent Foundation (to MGM), Novo Nordisk A/S (to MGM), ADA 1-19-PDF-099 (to PVS) and NIH DK122660 (to AHA).

\section{References}

Allison, Margaret B., Warren Pan, Alexander MacKenzie, Christa Patterson, Kimi Shah, Tammy Barnes, Wenwen Cheng, Alan Rupp, David P. Olson, and Martin G. Myers. 2018. "Defining the Transcriptional Targets of Leptin Reveals a Role for Atf3 in Leptin Action." Diabetes 67 (6): 1093-1104. https://doi.org/10.2337/db171395.

Bingham, Nathan C., Sunita Verma-Kurvari, Luis F. Parada, and Keith L. Parker. 2006. "Development of a Steroidogenic Factor 1/Cre Transgenic Mouse Line." Genesis 44 (9): 419-24. https://doi.org/10.1002/dvg.20231.

Campbell, John N., Evan Z. Macosko, Henning Fenselau, Tune H. Pers, Anna Lyubetskaya, Danielle Tenen, Melissa Goldman, et al. 2017. "A Molecular Census of Arcuate Hypothalamus and Median Eminence Cell Types." Nature Neuroscience 20 (3): 484-96. https://doi.org/10.1038/nn.4495.

Chen, Hong, Olga Charlat, Louis A Tartaglia, Elizabeth A Woolf, Xun Weng, Stephen J Ellis, Nathan D Lakey, et al. 1996. "Evidence That the Diabetes Gene Encodes 
the Leptin Receptor: Identification of a Mutation in the Leptin Receptor Gene in Db/Db Mice." Cell 84 (3): 491-95. https://doi.org/10.1016/S0092-8674(00)812945.

Chen, Renchao, Xiaoji Wu, Lan Jiang, and Yi Zhang. 2017. "Single-Cell RNA-Seq Reveals Hypothalamic Cell Diversity." Cell Reports 18 (13): 3227-41. https://doi.org/10.1016/j.celrep.2017.03.004.

Cheung, Clement C., Deborah M. Kurrasch, Jenna K. Liang, and Holly A. Ingraham. 2013. "Genetic Labeling of Steroidogenic Factor-1 (SF-1) Neurons in Mice Reveals Ventromedial Nucleus of the Hypothalamus (VMH) Circuitry Beginning at Neurogenesis and Development of a Separate Non-SF-1 Neuronal Cluster in the Ventrolateral VMH." Journal of Comparative Neurology 521 (6): 1268-88. https://doi.org/10.1002/cne.23226.

Dhillon, Harveen, Jeffrey M. Zigman, Chianping Ye, Charlotte E. Lee, Robert A. McGovern, Vinsee Tang, Christopher D. Kenny, et al. 2006. "Leptin Directly Activates SF1 Neurons in the VMH, and This Action by Leptin Is Required for Normal Body-Weight Homeostasis." Neuron 49 (2): 191-203. https://doi.org/10.1016/j.neuron.2005.12.021.

Dodd, Garron T., Amy A. Worth, Nicolas Nunn, Aaron K. Korpal, David A. Bechtold, Margaret B. Allison, Martin G. Myers, Michael A. Statnick, and Simon M. Luckman. 2014. "The Thermogenic Effect of Leptin Is Dependent on a Distinct Population of Prolactin-Releasing Peptide Neurons in the Dorsomedial Hypothalamus." Cell Metabolism 20 (4): 639-49. https://doi.org/10.1016/j.cmet.2014.07.022.

Elmquist, J. K., C. Bjørbaek, R. S. Ahima, J. S. Flier, and C. B. Saper. 1998. "Distributions of Leptin Receptor MRNA Isoforms in the Rat Brain." The Journal of Comparative Neurology 395 (4): 535-47.

Flak, Jonathan N., Paulette B. Goforth, James Dell'Orco, Paul V. Sabatini, Chien Li, Nadejda Bozadjieva, Matthew Sorensen, et al. 2020. "Ventromedial Hypothalamic Nucleus Neuronal Subset Regulates Blood Glucose Independently of Insulin." Journal of Clinical Investigation 130 (6): 2943-52. https://doi.org/10.1172/JCl134135.

Gavini, Chaitanya K., William C. Jones, and Colleen M. Novak. 2016. "Ventromedial Hypothalamic Melanocortin Receptor Activation: Regulation of Activity Energy Expenditure and Skeletal Muscle Thermogenesis: VMH and Skeletal Muscle Thermogenesis." The Journal of Physiology 594 (18): 5285-5301. https://doi.org/10.1113/JP272352.

Habib, Naomi, Inbal Avraham-Davidi, Anindita Basu, Tyler Burks, Karthik Shekhar, Matan Hofree, Sourav R Choudhury, et al. 2017. "Massively Parallel SingleNucleus RNA-Seq with DroNc-Seq." Nature Methods 14 (10): 955-58. https://doi.org/10.1038/nmeth.4407.

Hafemeister, Christoph, and Rahul Satija. 2019. "Normalization and Variance Stabilization of Single-Cell RNA-Seq Data Using Regularized Negative Binomial Regression." Genome Biology 20 (1): 296. https://doi.org/10.1186/s13059-0191874-1.

Hashikawa, Koichi, Yoshiko Hashikawa, Robin Tremblay, Jiaxing Zhang, James E Feng, Alexander Sabol, Walter T Piper, Hyosang Lee, Bernardo Rudy, and Dayu 
Lin. 2017. "Esr1+ Cells in the Ventromedial Hypothalamus Control Female Aggression." Nature Neuroscience 20 (11): 1580-90.

https://doi.org/10.1038/nn.4644.

Huo, Lihong, Kevin M. Gamber, Harvey J. Grill, and Christian Bjørbaek. 2008.

"Divergent Leptin Signaling in Proglucagon Neurons of the Nucleus of the Solitary Tract in Mice and Rats." Endocrinology 149 (2): 492-97. https://doi.org/10.1210/en.2007-0633.

Jones, Graham L., Gábor Wittmann, Eva B. Yokosawa, Hui Yu, Aaron J. Mercer, Ronald M. Lechan, and Malcolm J. Low. 2019. "Selective Restoration of Pomc Expression in Glutamatergic POMC Neurons: Evidence for a Dynamic Hypothalamic Neurotransmitter Network." Eneuro 6 (2): ENEURO.0400-18.2019. https://doi.org/10.1523/ENEURO.0400-18.2019.

Kim, Dong-Wook, Zizhen Yao, Lucas T. Graybuck, Tae Kyung Kim, Thuc Nghi Nguyen, Kimberly A. Smith, Olivia Fong, et al. 2019. "Multimodal Analysis of Cell Types in a Hypothalamic Node Controlling Social Behavior." Cell 179 (3): 713-728.e17. https://doi.org/10.1016/j.cell.2019.09.020.

Kim, Ki Woo, Jose Donato, Eric D. Berglund, Yun-Hee Choi, Daisuke Kohno, Carol F. Elias, Ronald A. Depinho, and Joel K. Elmquist. 2012. "FOXO1 in the Ventromedial Hypothalamus Regulates Energy Balance." The Journal of Clinical Investigation 122 (7): 2578-89. https://doi.org/10.1172/JCI62848.

Krashes, Michael J., Bhavik P. Shah, Joseph C. Madara, David P. Olson, David E. Strochlic, Alastair S. Garfield, Linh Vong, et al. 2014. "An Excitatory Paraventricular Nucleus to AgRP Neuron Circuit That Drives Hunger." Nature 507 (7491): 238-42. https://doi.org/10.1038/nature12956.

Krause, William C., and Holly A. Ingraham. 2017. "Origins and Functions of the Ventrolateral VMH: A Complex Neuronal Cluster Orchestrating Sex Differences in Metabolism and Behavior." In Sex and Gender Factors Affecting Metabolic Homeostasis, Diabetes and Obesity, edited by Franck Mauvais-Jarvis, 1043:199-213. Advances in Experimental Medicine and Biology. Cham: Springer International Publishing. https://doi.org/10.1007/978-3-319-70178-3_10.

Kunwar, Prabhat S., Moriel Zelikowsky, Ryan Remedios, Haijiang Cai, Melis Yilmaz, Markus Meister, and David J. Anderson. 2015. "Ventromedial Hypothalamic Neurons Control a Defensive Emotion State." ELife 4 (March). https://doi.org/10.7554/eLife.06633.

Kurrasch, D. M., C. C. Cheung, F. Y. Lee, P. V. Tran, K. Hata, and H. A. Ingraham. 2007. "The Neonatal Ventromedial Hypothalamus Transcriptome Reveals Novel Markers with Spatially Distinct Patterning." Journal of Neuroscience 27 (50): 13624-34. https://doi.org/10.1523/JNEUROSCI.2858-07.2007.

Lam, Brian Y.H., Irene Cimino, Joseph Polex-Wolf, Sara Nicole Kohnke, Debra Rimmington, Valentine lyemere, Nicholas Heeley, et al. 2017. "Heterogeneity of Hypothalamic Pro-Opiomelanocortin-Expressing Neurons Revealed by SingleCell RNA Sequencing." Molecular Metabolism 6 (5): 383-92. https://doi.org/10.1016/j.molmet.2017.02.007.

Lee, Hyosang, Dong-Wook Kim, Ryan Remedios, Todd E. Anthony, Angela Chang, Linda Madisen, Hongkui Zeng, and David J. Anderson. 2014. "Scalable Control 
of Mounting and Attack by Esr1+ Neurons in the Ventromedial Hypothalamus." Nature 509 (7502): 627-32. https://doi.org/10.1038/nature13169.

Leshan, Rebecca L., Marie Björnholm, Heike Münzberg, and Martin G. Myers. 2006. "Leptin Receptor Signaling and Action in the Central Nervous System." Obesity 14 (August): 208S-212S. https://doi.org/10.1038/oby.2006.310.

Lindberg, Daniel, Peilin Chen, and Chien Li. 2013. "Conditional Viral Tracing Reveals That Steroidogenic Factor 1-Positive Neurons of the Dorsomedial Subdivision of the Ventromedial Hypothalamus Project to Autonomic Centers of the Hypothalamus and Hindbrain." The Journal of Comparative Neurology 521 (14): 3167-90. https://doi.org/10.1002/cne.23338.

Lun, Aaron T.L., Davis J. McCarthy, and John C. Marioni. 2016. "A Step-by-Step

Workflow for Low-Level Analysis of Single-Cell RNA-Seq Data with

Bioconductor." F1000Research 5 (October): 2122. https://doi.org/10.12688/f1000research.9501.2.

Meek, Thomas H., Miles E. Matsen, Mauricio D. Dorfman, Stephan J. Guyenet, Vincent Damian, Hong T. Nguyen, Gerald J. Taborsky, and Gregory J. Morton. 2013. "Leptin Action in the Ventromedial Hypothalamic Nucleus Is Sufficient, but Not Necessary, to Normalize Diabetic Hyperglycemia." Endocrinology 154 (9): 306776. https://doi.org/10.1210/en.2013-1328.

Meek, Thomas H., Jarrell T. Nelson, Miles E. Matsen, Mauricio D. Dorfman, Stephan J. Guyenet, Vincent Damian, Margaret B. Allison, et al. 2016. "Functional Identification of a Neurocircuit Regulating Blood Glucose." Proceedings of the National Academy of Sciences of the United States of America 113 (14): E20732082. https://doi.org/10.1073/pnas.1521160113.

Minokoshi, Y., M. S. Haque, and T. Shimazu. 1999. "Microinjection of Leptin into the Ventromedial Hypothalamus Increases Glucose Uptake in Peripheral Tissues in Rats." Diabetes 48 (2): 287-91. https://doi.org/10.2337/diabetes.48.2.287.

Noble, Emily E., Charles J. Billington, Catherine M. Kotz, and ChuanFeng Wang. 2014. "Oxytocin in the Ventromedial Hypothalamic Nucleus Reduces Feeding and Acutely Increases Energy Expenditure." American Journal of PhysiologyRegulatory, Integrative and Comparative Physiology 307 (6): R737-45. https://doi.org/10.1152/ajpregu.00118.2014.

Persson-Augner, David, Yong-Woo Lee, Sulay Tovar, Carlos Dieguez, and Björn Meister. 2014. "Delta-Like 1 Homologue (DLK1) Protein in Neurons of the Arcuate Nucleus That Control Weight Homeostasis and Effect of Fasting on Hypothalamic DLK1 MRNA." Neuroendocrinology 100 (2-3): 209-20. https://doi.org/10.1159/000369069.

Rousso, David L., Mu Qiao, Ruth D. Kagan, Masahito Yamagata, Richard D. Palmiter, and Joshua R. Sanes. 2016. "Two Pairs of ON and OFF Retinal Ganglion Cells Are Defined by Intersectional Patterns of Transcription Factor Expression." Cell Reports 15 (9): 1930-44. https://doi.org/10.1016/j.celrep.2016.04.069.

Sabatini, Paul V, Jine Wang, Alan C Rupp, Alison H Affinati, Jonathan N Flak, Chien Li, David P Olson, and Martin G Myers. 2021. "TTARGIT AAVs Mediate the Sensitive and Flexible Manipulation of Intersectional Neuronal Populations in Mice." ELife 10 (March): e66835. https://doi.org/10.7554/eLife.66835. 
Tartaglia, Louis A., Marlene Dembski, Xun Weng, Nanhua Deng, Janice Culpepper, Rene Devos, Grayson J. Richards, et al. 1995. "Identification and Expression Cloning of a Leptin Receptor, OB-R." Cell 83 (7): 1263-71. https://doi.org/10.1016/0092-8674(95)90151-5.

Timshel, Pascal N, Jonatan J Thompson, and Tune H Pers. 2020. "Genetic Mapping of Etiologic Brain Cell Types for Obesity." ELife 9 (September): e55851. https://doi.org/10.7554/eLife.55851.

Toda, C., T. Shiuchi, H. Kageyama, S. Okamoto, E. A. Coutinho, T. Sato, Y. OkamatsuOgura, et al. 2013. "Extracellular Signal-Regulated Kinase in the Ventromedial Hypothalamus Mediates Leptin-Induced Glucose Uptake in Red-Type Skeletal Muscle." Diabetes 62 (7): 2295-2307. https://doi.org/10.2337/db12-1629.

Vander Tuig, Jerry G., Allen W. Knehans, and Dale R. Romsos. 1982. "Reduced Sympathetic Nervous System Activity in Rats with Ventromedial Hypothalamic Lesions." Life Sciences 30 (11): 913-20. https://doi.org/10.1016/00243205(82)90619-1.

Vong, Linh, Chianping Ye, Zongfang Yang, Brian Choi, Streamson Chua, and Bradford B. Lowell. 2011. "Leptin Action on GABAergic Neurons Prevents Obesity and Reduces Inhibitory Tone to POMC Neurons." Neuron 71 (1): 142-54. https://doi.org/10.1016/j.neuron.2011.05.028.

Wolock, Samuel L., Romain Lopez, and Allon M. Klein. 2019. "Scrublet: Computational Identification of Cell Doublets in Single-Cell Transcriptomic Data." Cell Systems 8 (4): 281-291.e9. https://doi.org/10.1016/j.cels.2018.11.005.

\section{Figure legends}

Figure 1: Identification of VMH neurons from mice. (A) Schematic of VMH nuclei isolation and single-cell sequencing protocol. (B) UMAP projection of 21,585 neuronal nuclei colored and labeled by cluster designation. (C) Expression profile of the top enriched genes for each cluster (colored on bottom), including GABAergic (Gad1) and glutamatergic (Slc17a6) markers. (D) Expression of Nr5a1 and Fezf1 in individual cells in UMAP space. (E) Nr5a1-Cre TRAP-seq overview. Nr5a1-Cre mice were crossed with ROSA26 ${ }^{\text {EGFP-L10a }}$ mice, resulting in VMH-restricted eGFP-L10a expression. Representative image shows GFP-IR (black) in a coronal section from these mice. (F) TRAP-seq revealed the enrichment of thousands of genes (including Nr5a1 and Fezf1) in these cells relative to non-TRAP material. (G) Expression profile of the top enriched genes from Nr5a1-Cre TRAP-seq across clusters; gray box indicates presumptive VMH cells. (H) Magnitude of the first principal component after performing principal components analysis for the genes enriched in Nr5a1-Cre TRAP-seq. (I) Venn diagram of genes enriched in Nr5a1-Cre TRAP-seq (TRAP enriched), in snRNA-seq VMH 
pseudo-TRAP (pseudo-TRAP enriched), or both (Common). Number in parentheses refers to the number of genes in each category. (J) Histograms of expression level for genes by enrichment geneset in each dataset (Nr5a1-Cre TRAP-seq or snRNA-seq). (K) Mean scaled expression for each cluster for the top genes enriched in Nr5a1-Cre TRAP-seq.

Figure 2: VMH neuronal populations can be grouped into 6 major classes. (A) UMAP projection of 6,049 VMH neurons colored and labeled by cluster designation. (B) Prevalence of clusters across samples, mean \pm SEM. (C) Expression profile of the top enriched genes for each cluster. (D) Hierarchical clustering and mean expression of marker genes for each class of neurons. (E) ES $\mu$ for the top 3 marker genes for each population determined by CELLEX. (F) Median maximal pairwise expression correlation for each cut of the hierarchical tree resulting in 2-24 clusters. (G) Percent of cells in each VMH cluster that correspond to each neuronal cluster (from Figure 1). (H) ES $\mu$ for the top 5 marker genes for each major class determined by CELLEX.

Figure 3: VMH ${ }^{\text {Lepr }}$ neurons represent a distinct class of VMH neurons. (A) Diagram of strategy to transcriptionally profile the $\mathrm{VMH}^{\mathrm{Lepr}}$ neurons by crossing Lepr ${ }^{\mathrm{Cre}}$ and Slc17a6 ${ }^{F l p o}$ to a mouse line in which the ROSA26 (R26) locus contains a CAG-driven, Flp- and Cre-dependent eGFP:L10a allele (RCFL ${ }^{\text {eGFP-L10a }}$ ). (below) A representative image of GFP-IR (black) expression in Lepr ${ }^{C r e} ;$ Slc17a6 ${ }^{\text {Flpo; }} ; R C F L^{\text {eGFP-L10a }}$ (Lepr $^{\text {Slc17a6 }}$ L10a) mice. (B) Scaled counts per million (CPM) for each gene in Lepr ${ }^{\text {Slc17a6 }}{ }_{-L 10 a}$ mice. (C) Expression and enrichment of genes from Lepr ${ }^{\text {Slc17a6 }}$ L10a VMH pulldown. (D) Expression of Lepr in individual VMH neurons in UMAP space. (E) Magnitude of the first principal component after performing principal components analysis for the genes enriched in Lepr ${ }^{\text {Slc17a6 }}$-L10a VMH TRAP-seq, projected into UMAP space. (F) Mean class expression (left), Lepr cluster expression (center), and Lepr ${ }^{\text {Slc17a6 }}$-L10a TRAP-seq enrichment (right) of the top genes unique to the $\mathrm{VMH}^{\mathrm{Lepr}}$ population by both TRAP and pseudo-TRAP. (G-I) Sagittal Allen Brain Atlas in situ images for (G) Gpr149, (H) Rai14, and (I) Tnfrsf8; all probes shown in black. 
Figure 4: Macaque VMH populations revealed by snRNA-seq. (A) Schematic of experimental process for macaque snRNA-seq. (B) UMAP projection of 3,752 VMH neuronal nuclei colored and labeled by cluster designation. (C) Expression profile of the top enriched genes for each cluster. (D) Violin plot of normalized expression for marker genes for each $\mathrm{VMH}$ neuronal population. (E) ES $\mu$ for the top 5 marker genes for each cluster determined by CELLEX. (F) Pairwise scaled expression correlation (Pearson's $r$ ) for each macaque and mouse VMH neuronal cluster.

Figure 5: VMH populations are conserved between mouse and macaque. (A-B) Mouse and macaque snRNA-seq datasets were $(\mathbf{A})$ merged using canonical correlation analysis and (B) projected onto UMAP space, colored here by species. (C) UMAP projection of VMH neuronal nuclei colored and labeled by cluster designation. (D) Mean scaled expression of marker genes across integrated clusters by species. (E) Proportion of cells in each cluster from the sample for each species (mean \pm SEM). (F) Expression profile of the top enriched genes for each cluster. (G) Mapping of species-specific clusters onto the integrated clusters. (H) Species-specific ES $\mu$ for the top 3 marker genes for each integrated cluster determined by CELLEX. 


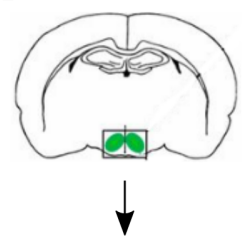

\section{Neurons} 21,585 nuclei

VMH dissection

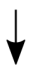

nuclei isolation<smiles>[C]1CCCC1</smiles>

$10 x$ sequencing 26

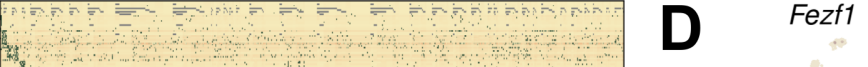

Gad1

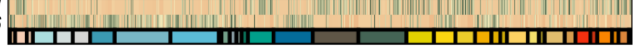

Scaled

$\begin{array}{lllll}\text { expression }^{-1} & 0 & 1 & 2 & 3\end{array}$

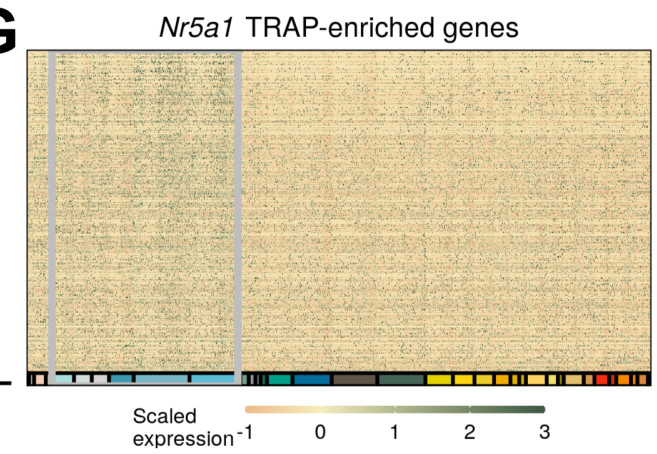

Nr5a1

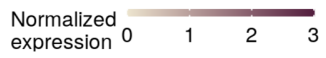

H

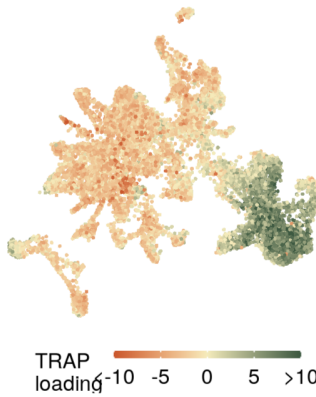

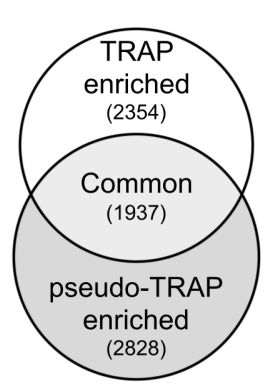

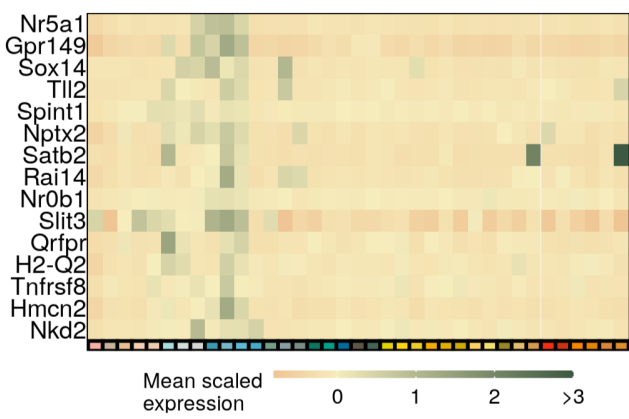

G
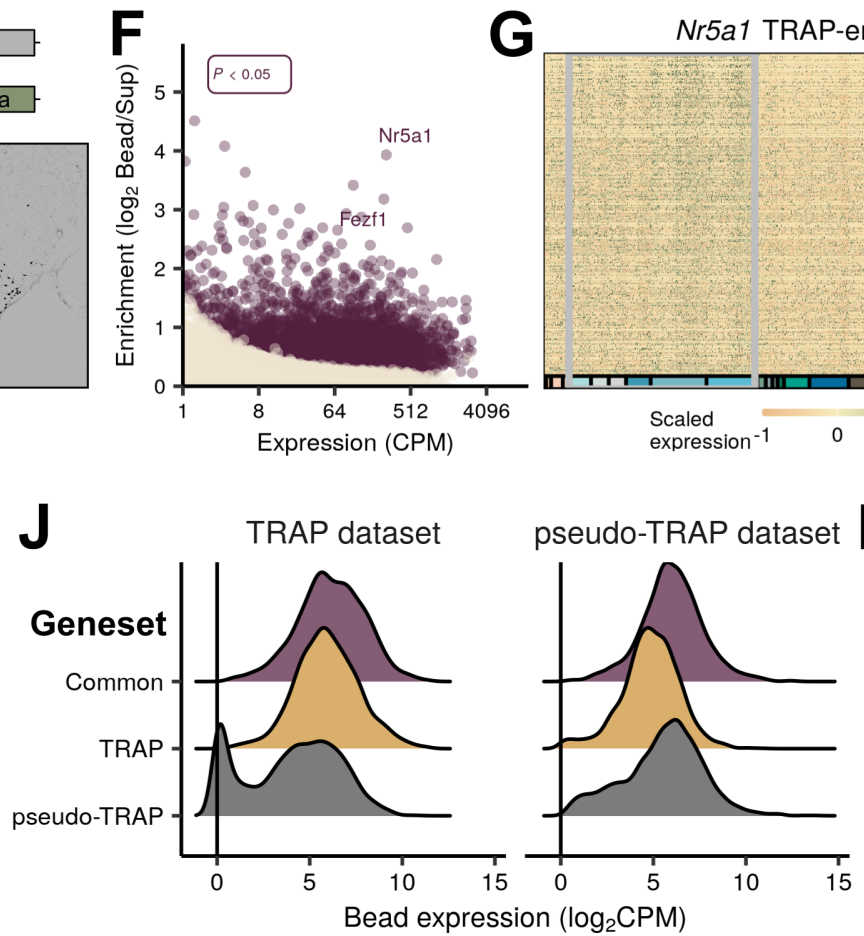

.




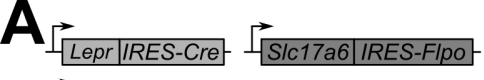

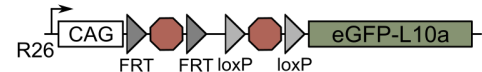

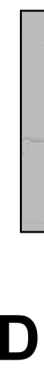

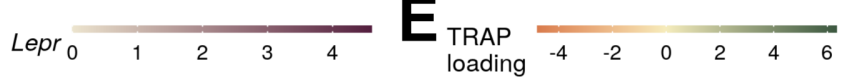

E
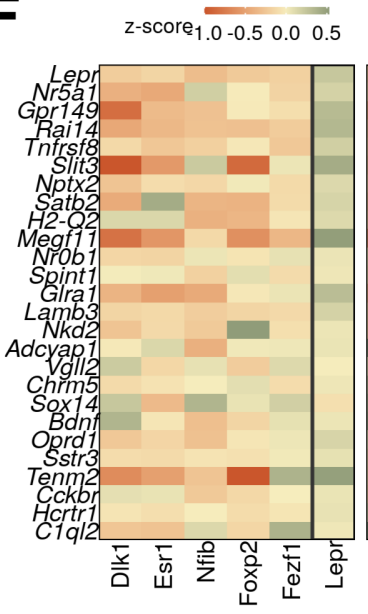

B

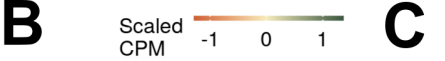
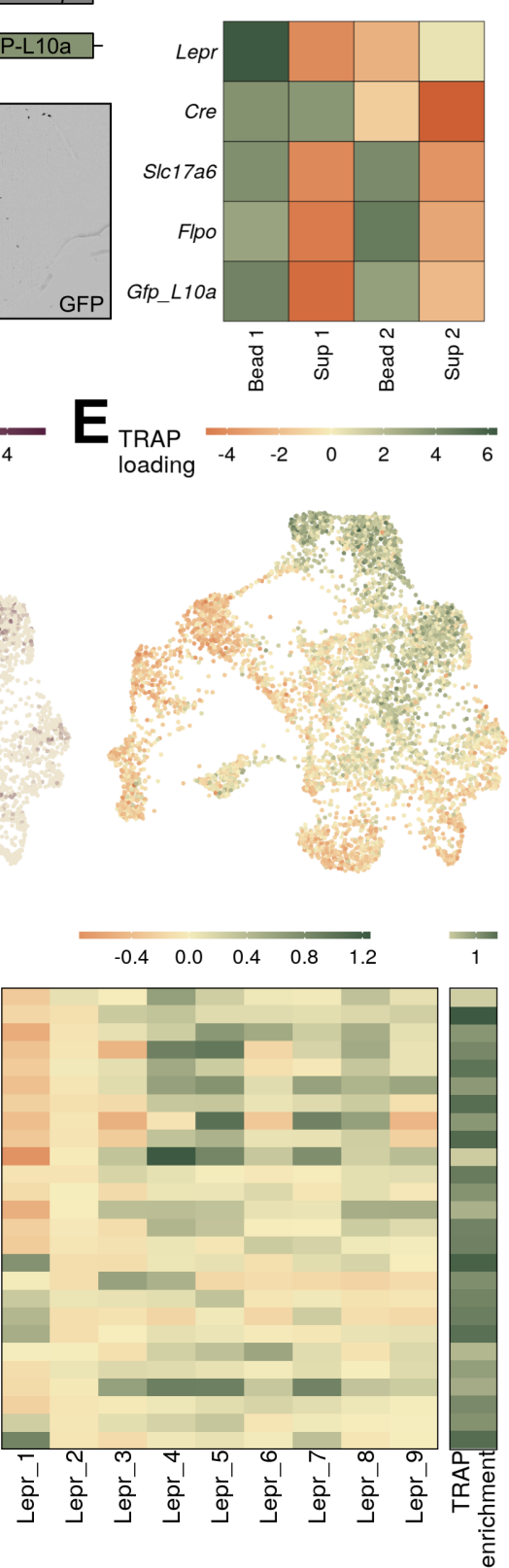
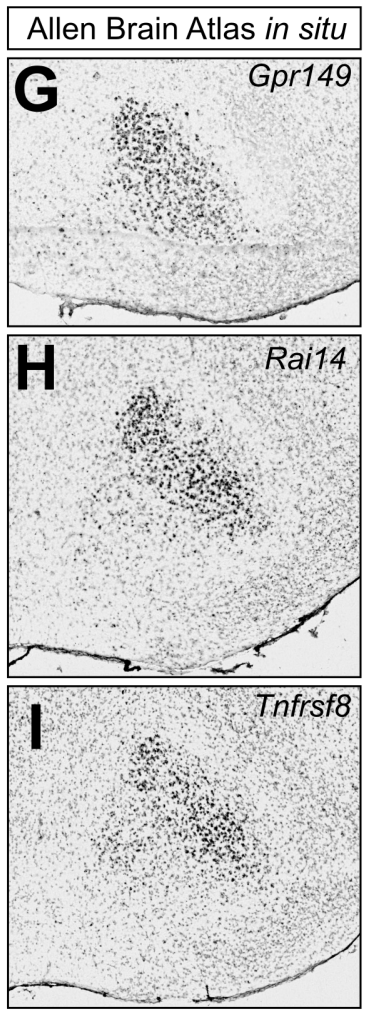
A

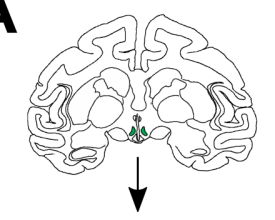

VMH dissection

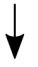

nuclei isolation

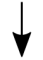

$10 x$ sequencing
B

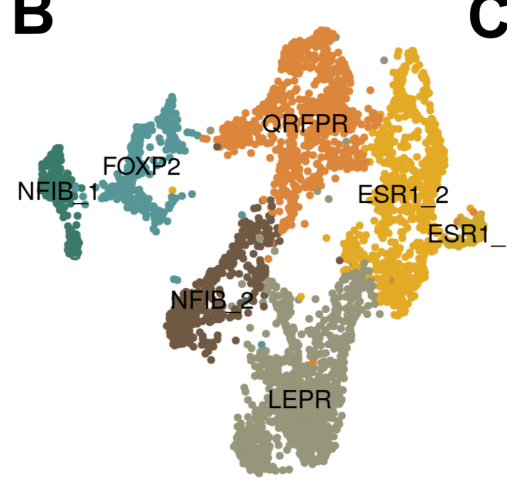

$\mathrm{ESH}_{0.0} \quad 0.2 \quad 0.4 \quad 0.6 \quad 0.8$

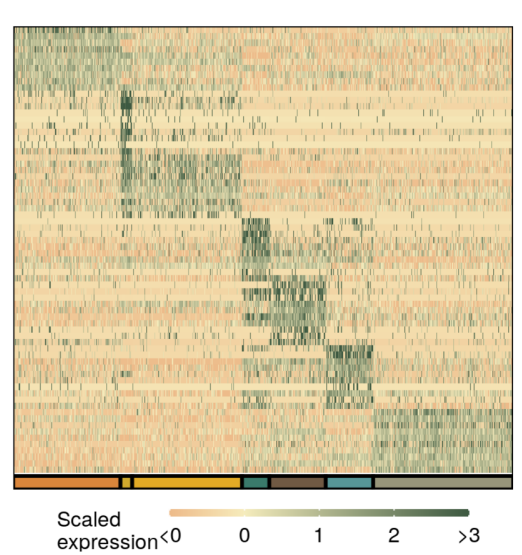

$\mathbf{F}$
D

QRFPR
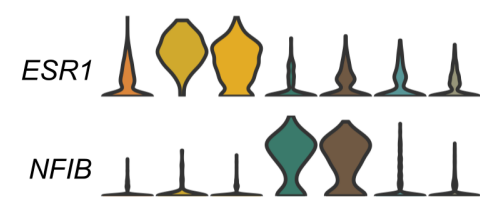

FOXP2 $1, \perp \perp \perp\} 1$

LEPR

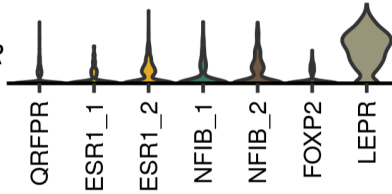

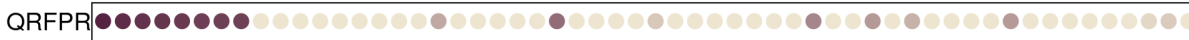
ESR1_1 ESR1_2 $000000000000 \bullet \bullet \bullet \bullet \bullet \bullet \bullet \bullet \bullet \bullet ० 0000000000000000000000000000000$ NFIB_1

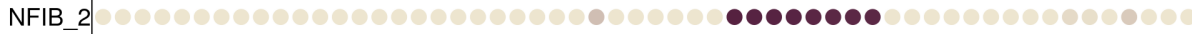
FOXP2 $00000000000000000000000000 \bullet 000 \bullet 00000000 \bullet \bullet \bullet \bullet \bullet \bullet \bullet \bullet ० 000000$ ( LEPR $00000000000000000000000000000000000000000000000 \bullet \bullet \bullet \bullet \bullet \bullet \bullet \bullet$

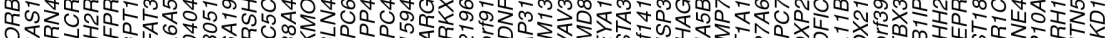

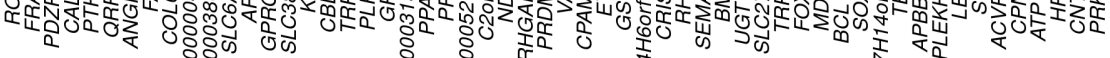

QRFPR ESR1_1 ESR1_2 NFIB_1 NFIB_2 FOXP2 LEPR

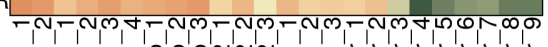
ET-

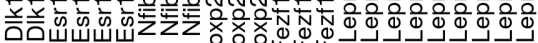
$\begin{array}{llllll}\text { Pearson's r } & -0.1 & 0.0 & 0.1 & 0.2 & 0.3\end{array}$ 

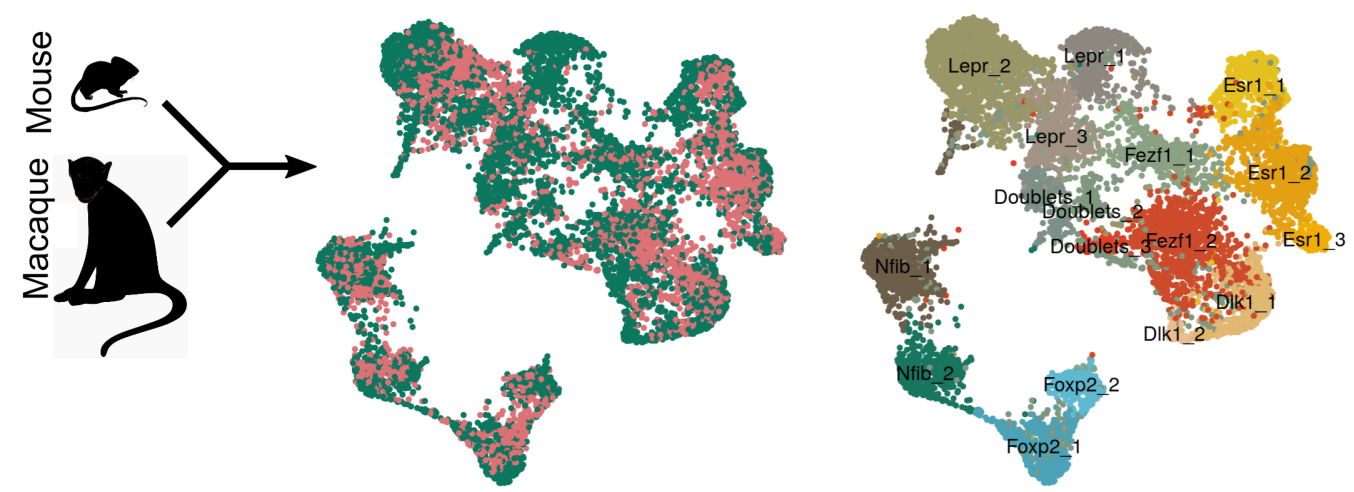

E

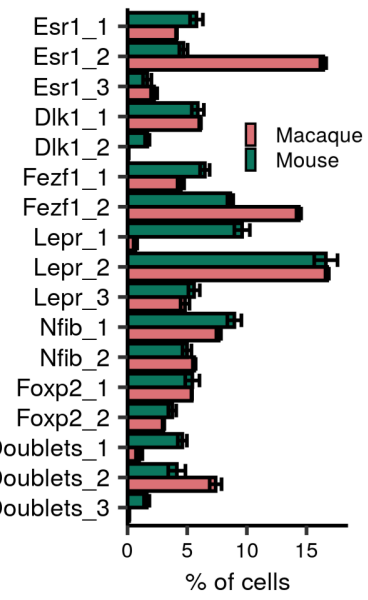

G

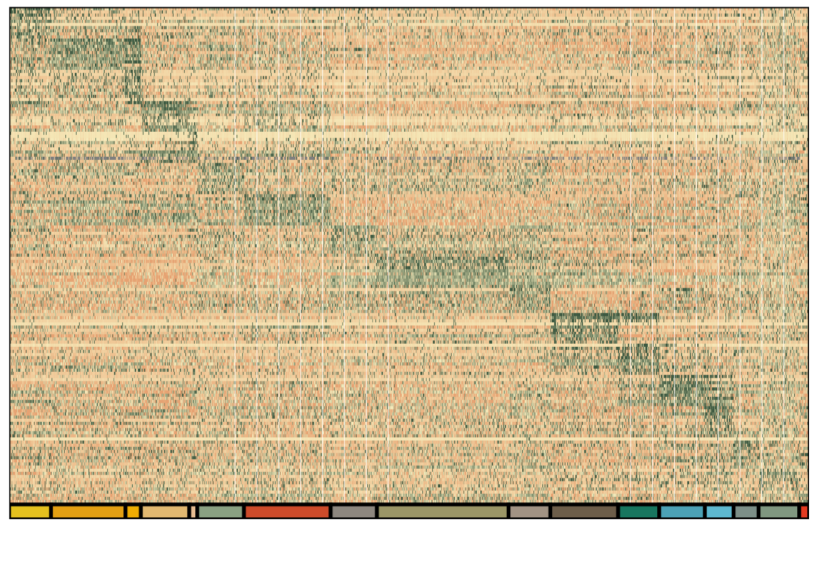

D
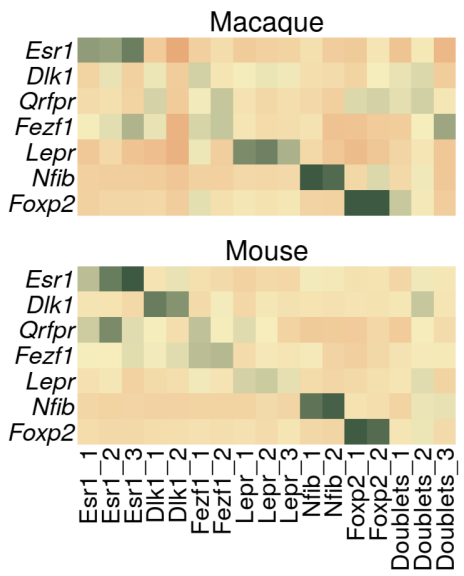

Mouse

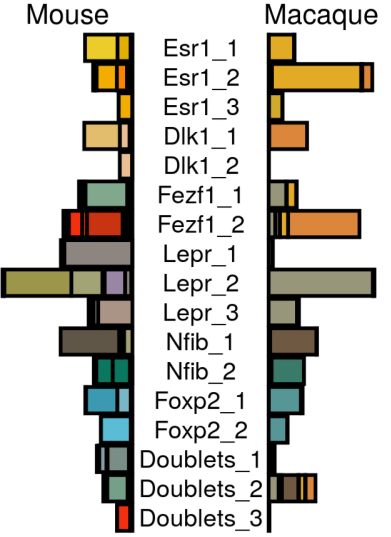

Esr1_1-

Esr1_2 Dop

Esr1_3

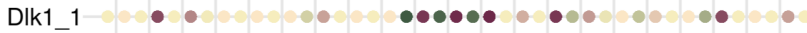

Dlk1_2 -

Fezf1_1-

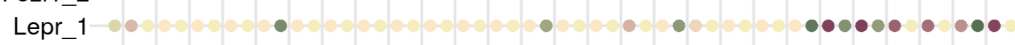

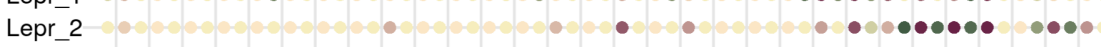

Lepr_3 -

Nfib_1

Nfib_2

Foxp2_1

Foxp2_2 Doublets_1

Doublets_2

Doublets_3

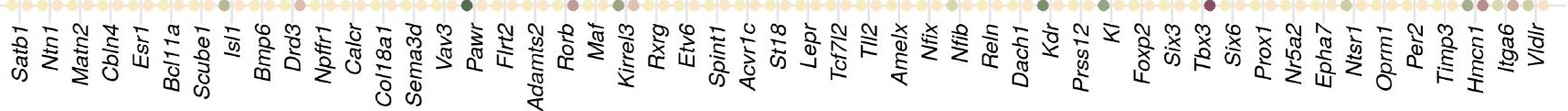




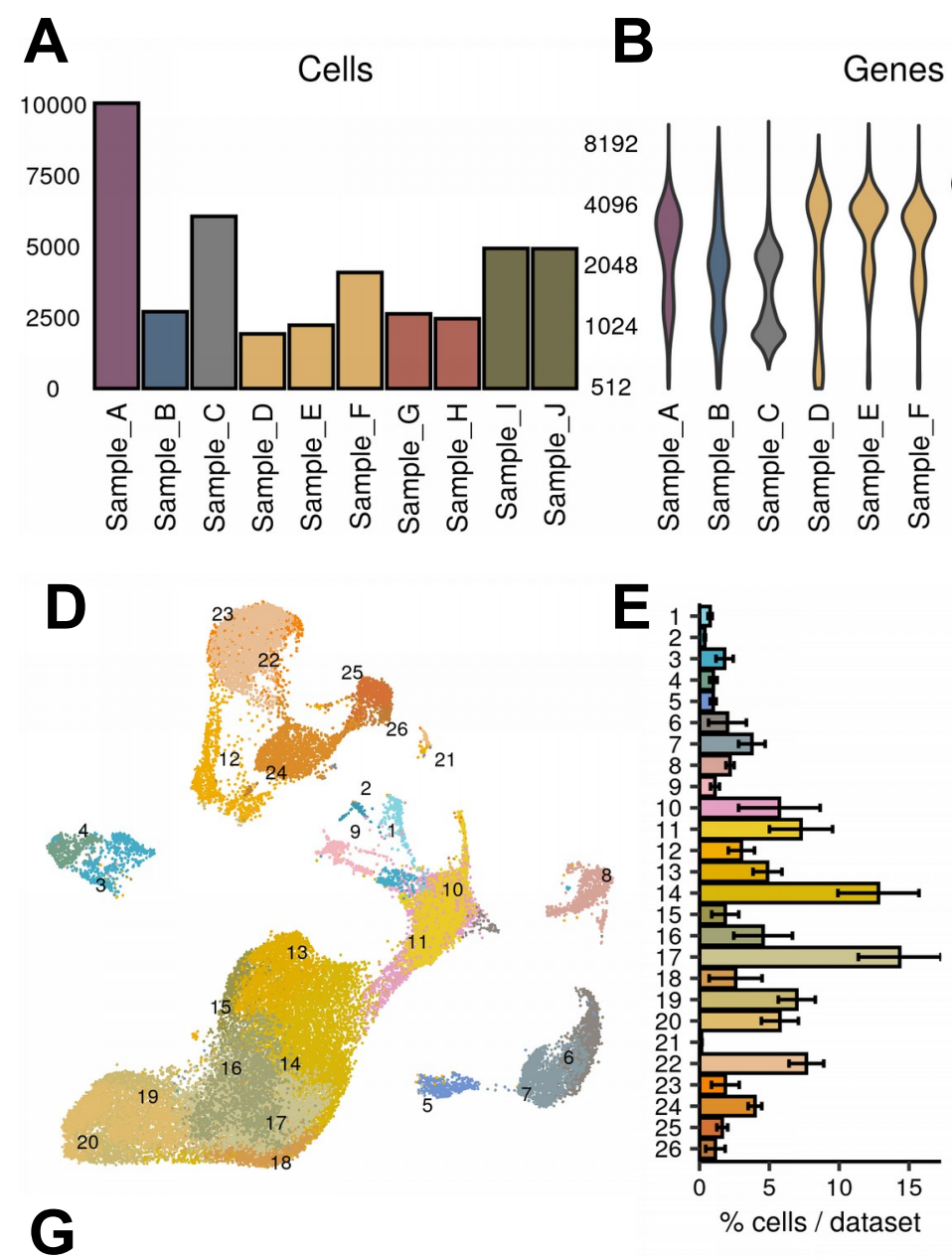

C
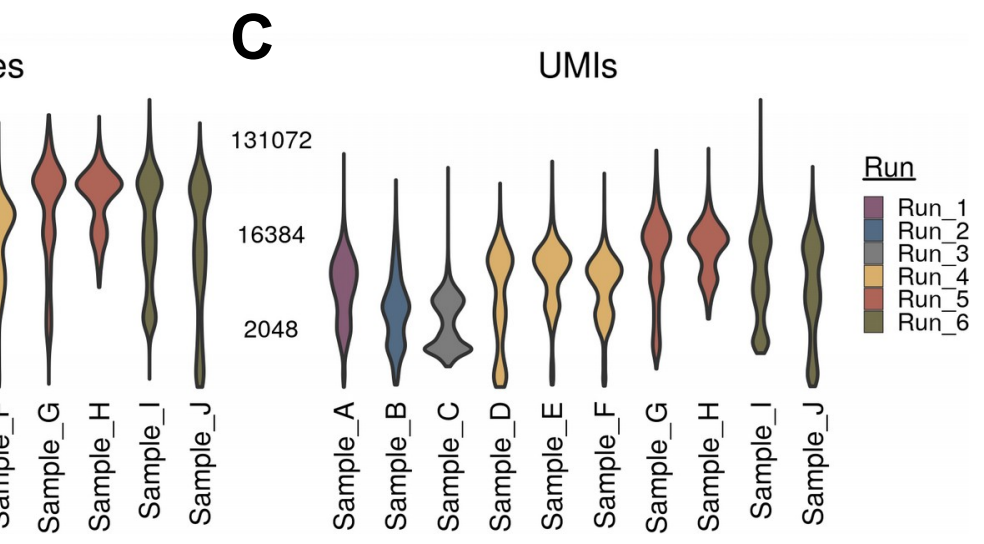

$F_{v t n}\left\lfloor\nabla_{\perp 111} \perp 11,1,1,1,11111\right.$

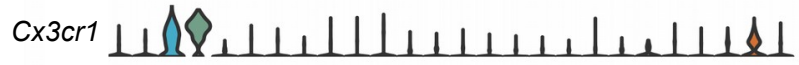

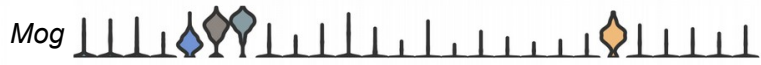
Pdgfra $\perp \perp, 1 \Omega \Omega l 11,1,11,1,1111$

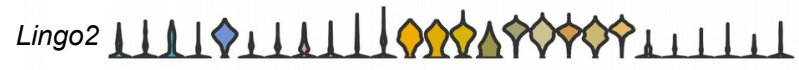

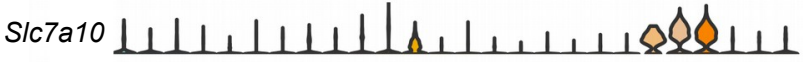
Tshr L.1111ل11111,1111,11/1

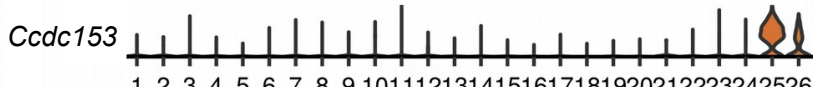
1234567891011121314151617181920212223242526
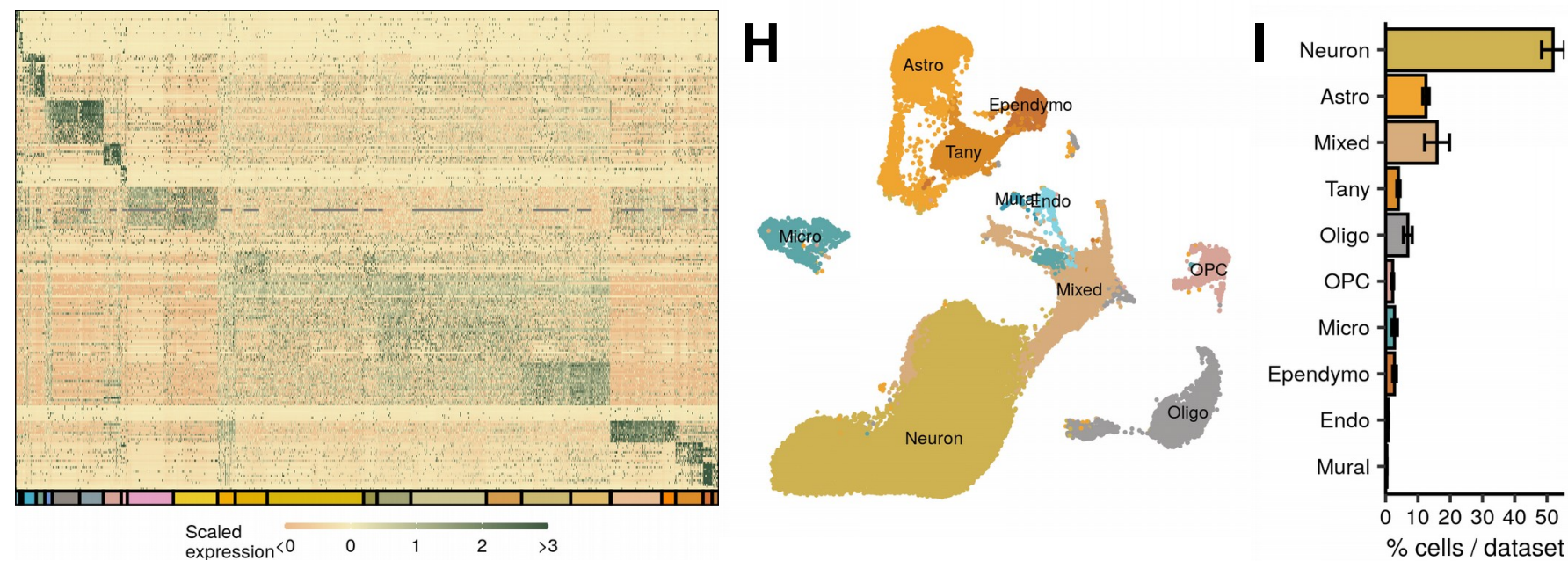

Figure S1: Mouse snRNA-seq identifies major CNS classes. (A-C) The number of (A) cells, (B) genes, and (C) UMls detected per sample used in this study after quality control. (D) UMAP projection of all 42,040 cells, colored by cluster. (E) Average percent of cells in each cluster across all samples. (F) Representative marker genes for each of the major CNS cell types. (G) Expression profile of top enriched genes for each cluster. (H) UMAP representation of cell type classification. (I) Quantification of cell classes per sample. 


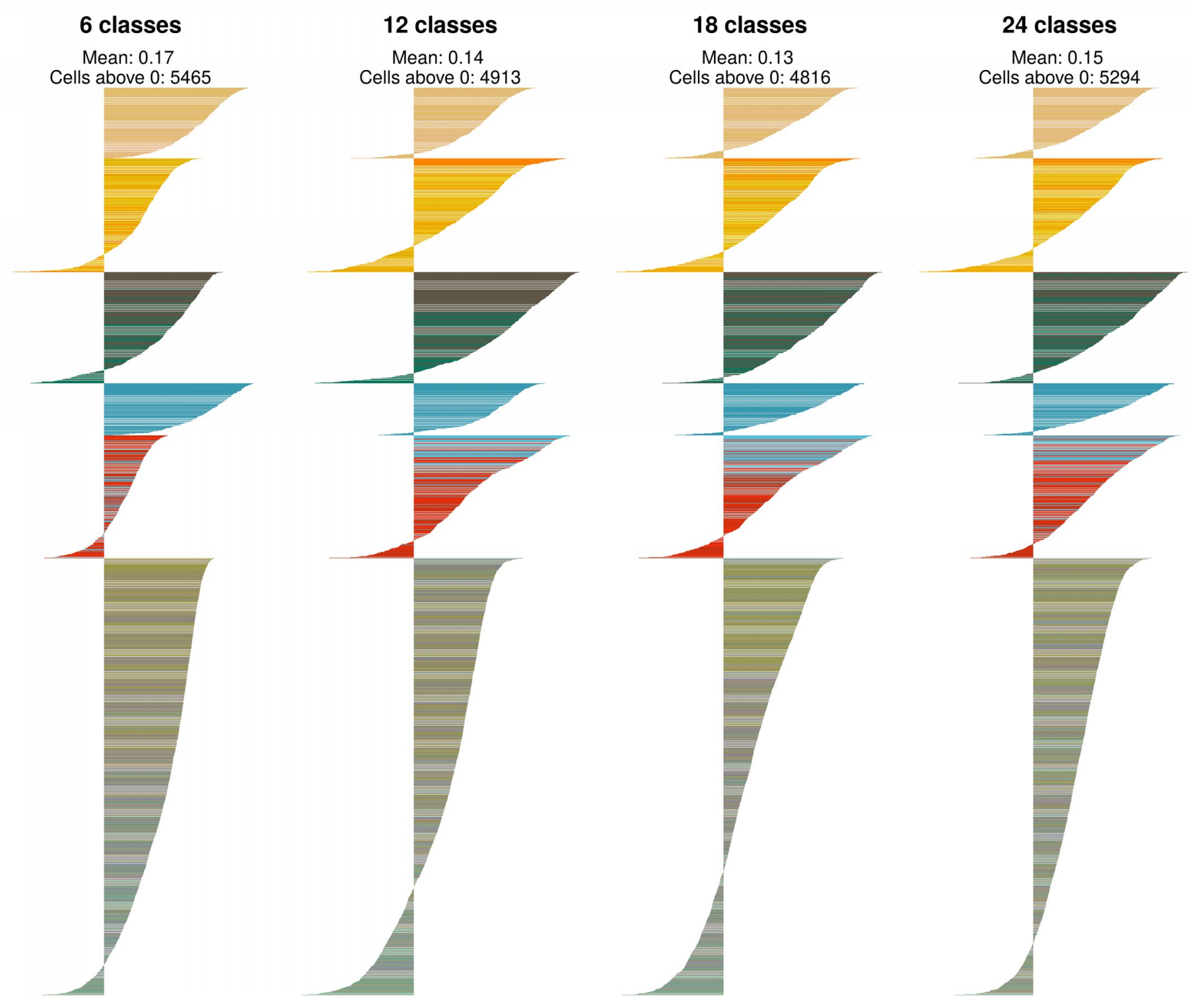

Figure S2: Identification of VMH neuronal "classes." The silhouette width for each cell for each level of VMH neuron classification. The mean silhouette width and the number of cells with a silhouette width greater than 0 are noted above the plot. Cells are colored by their cluster color in Fig. 2A. 


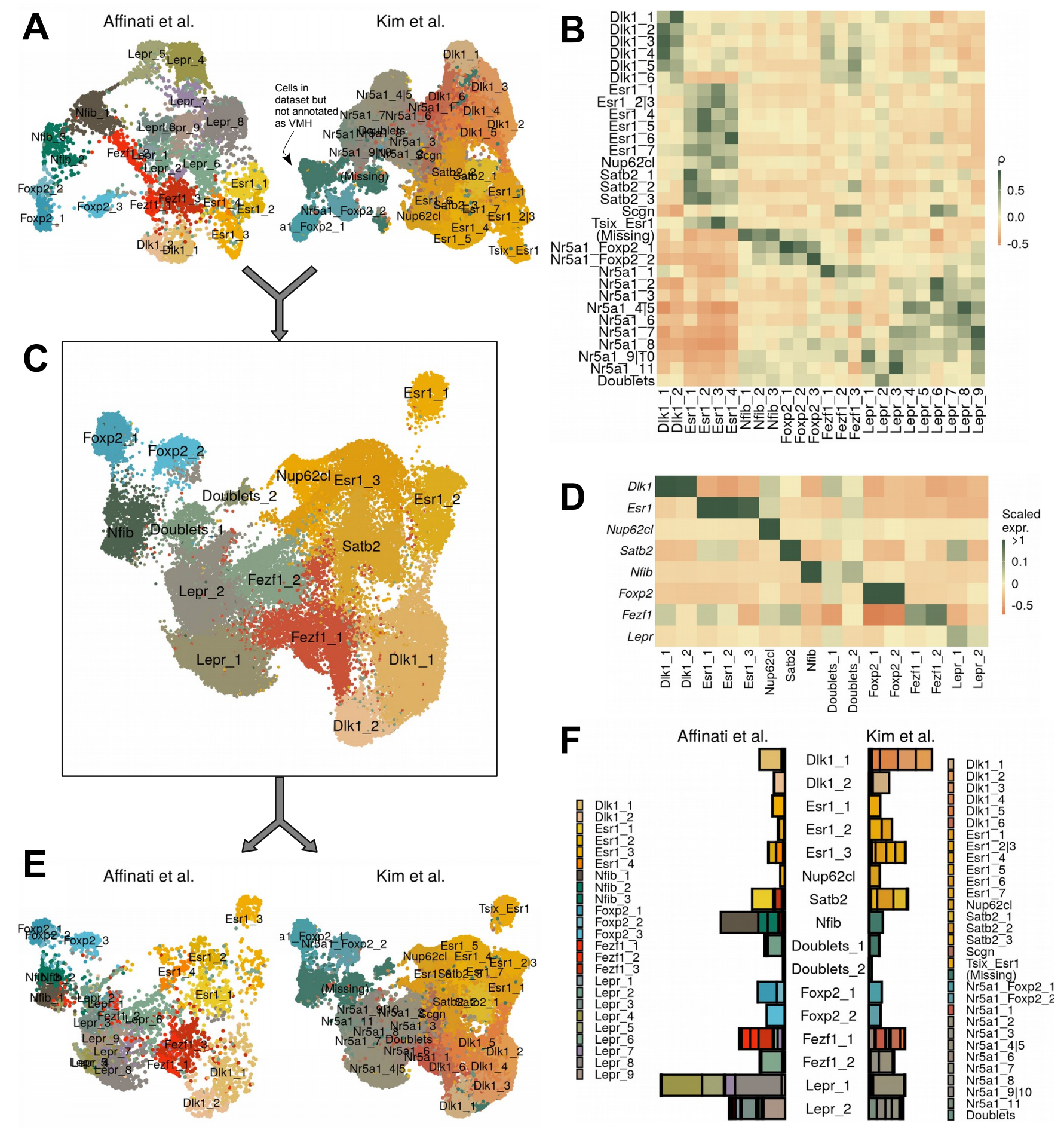

Figure S3: Comparison with VMH data from Kim et al. (A) UMAP projection of each dataset separately. Cells labeled "(Missing)" were present in the dataset, but were excluded from final VMH clustering. (B) Pair-wise expression correlation of variable genes for each cluster in each dataset. (C) UMAP projection of CCA-integrated data, colored by cluster. (D) Mean scaled expression of marker genes for each cluster. (E) UMAP projection of integrated data, by dataset of origin. (F) Breakdown of cluster designation from original dataset and integrated dataset. 


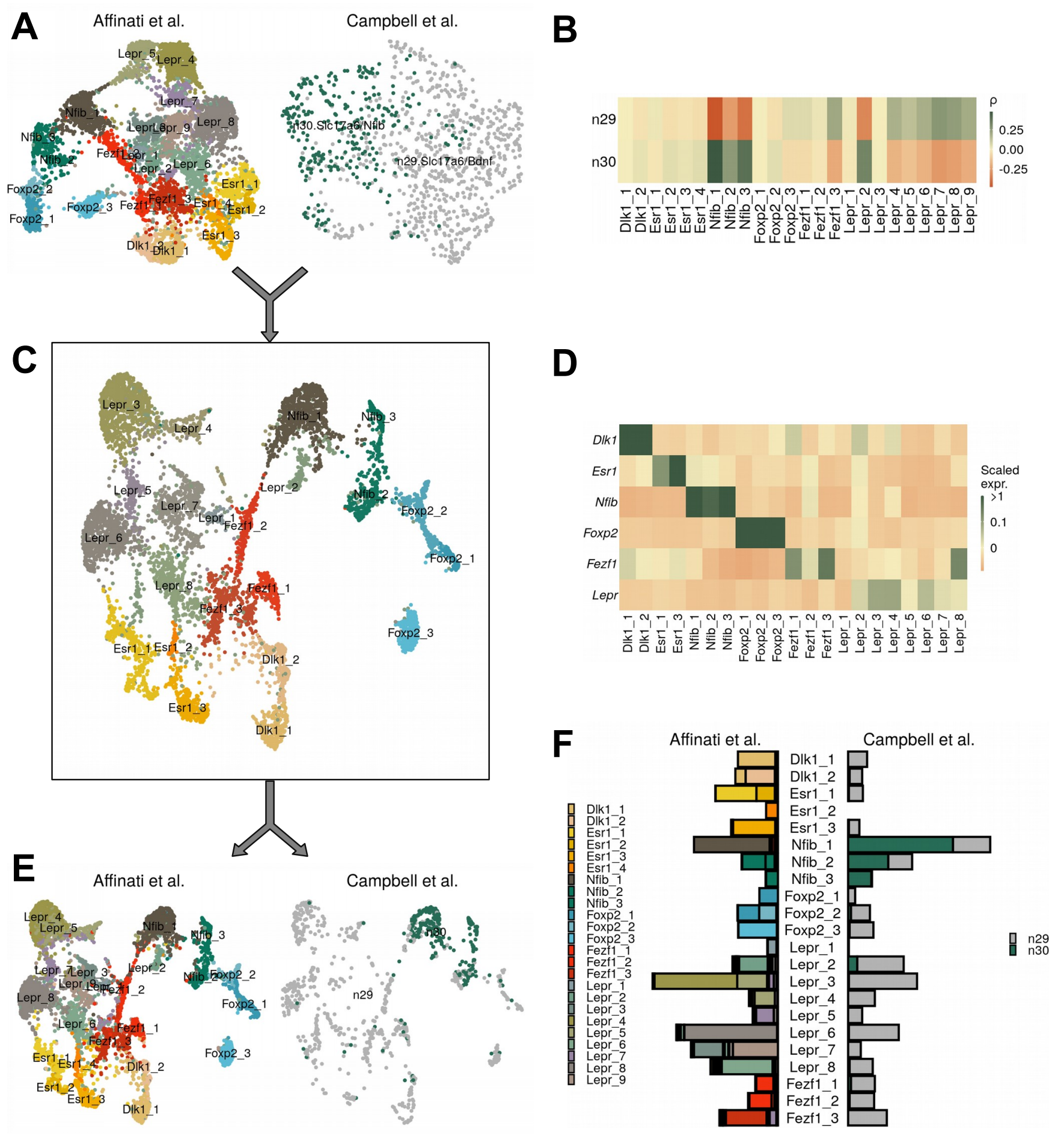

Figure S4: Comparison of data with VMH data from Campbell et al. (A) UMAP projection of each dataset separately. (B) Pair-wise expression correlation of variable genes for each cluster in each dataset. (C) UMAP projection of CCA-integrated data, colored by cluster. (D) Mean scaled expression of marker genes for each cluster. (E) UMAP projection of integrated data, by dataset of origin. (F) Breakdown of cluster designation from original dataset and integrated dataset. 
A All cells

Nfib
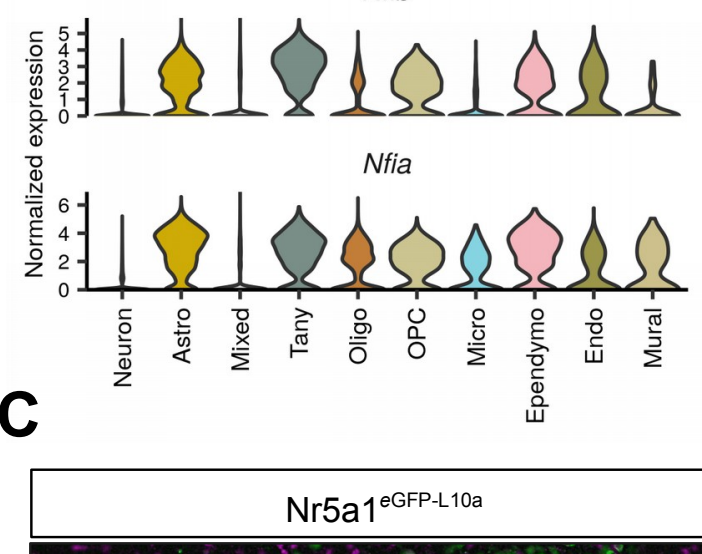

B

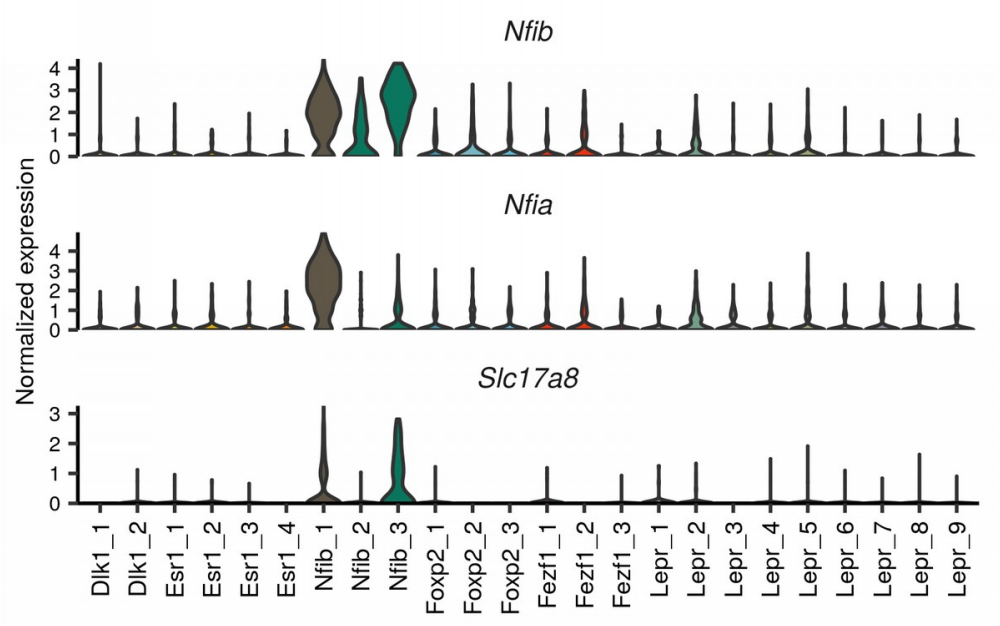

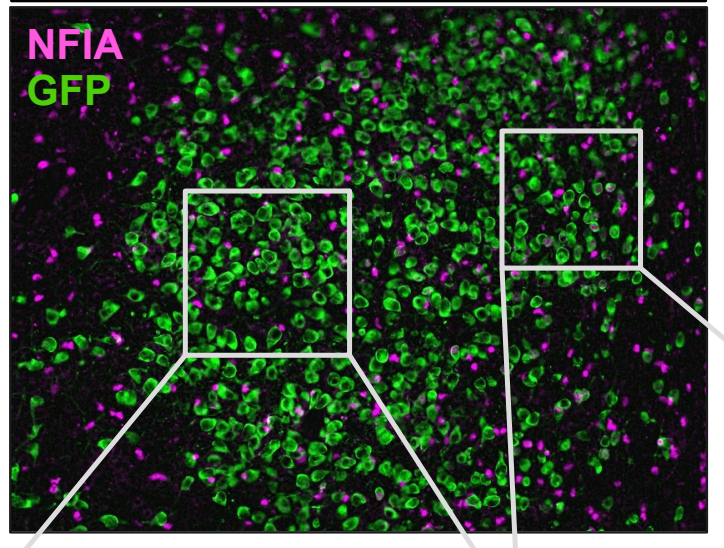
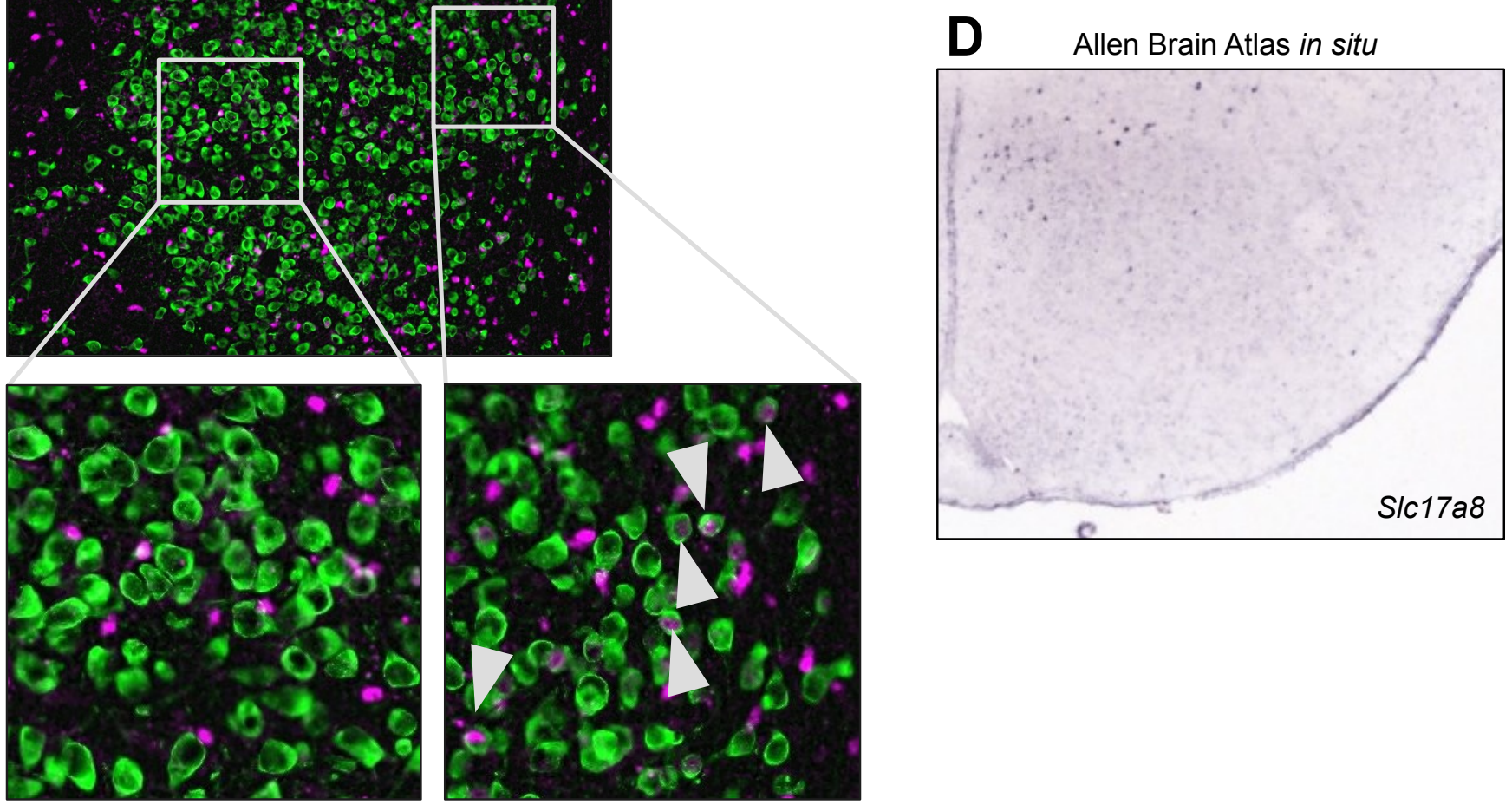

Figure S5: VMH Nfib population localizes to dorsomedial compartment. (A-B) Nfia marks (B) a subset of $\mathrm{VMH}^{\mathrm{Nib}}$ neurons but is also widely expressed in $(\mathbf{A})$ non-neuronal populations in the VMH. (C) Representative image showing GFP-IR (green) and NFIA-IR (magenta) in Nr5a1 ${ }^{\text {eGFP-L10a }}$ (Nr5a1-Cre;R26 ${ }^{\text {GFP-L10a }}$ ) mice; white arrowheads indicate colocalization. (B) Slc17a8 is a marker for $\mathrm{VMH}^{\mathrm{Nib}}$ cells and (D) is expressed in the most dorosmedial VMH according to the Allen Brain Atlas in situ database. 


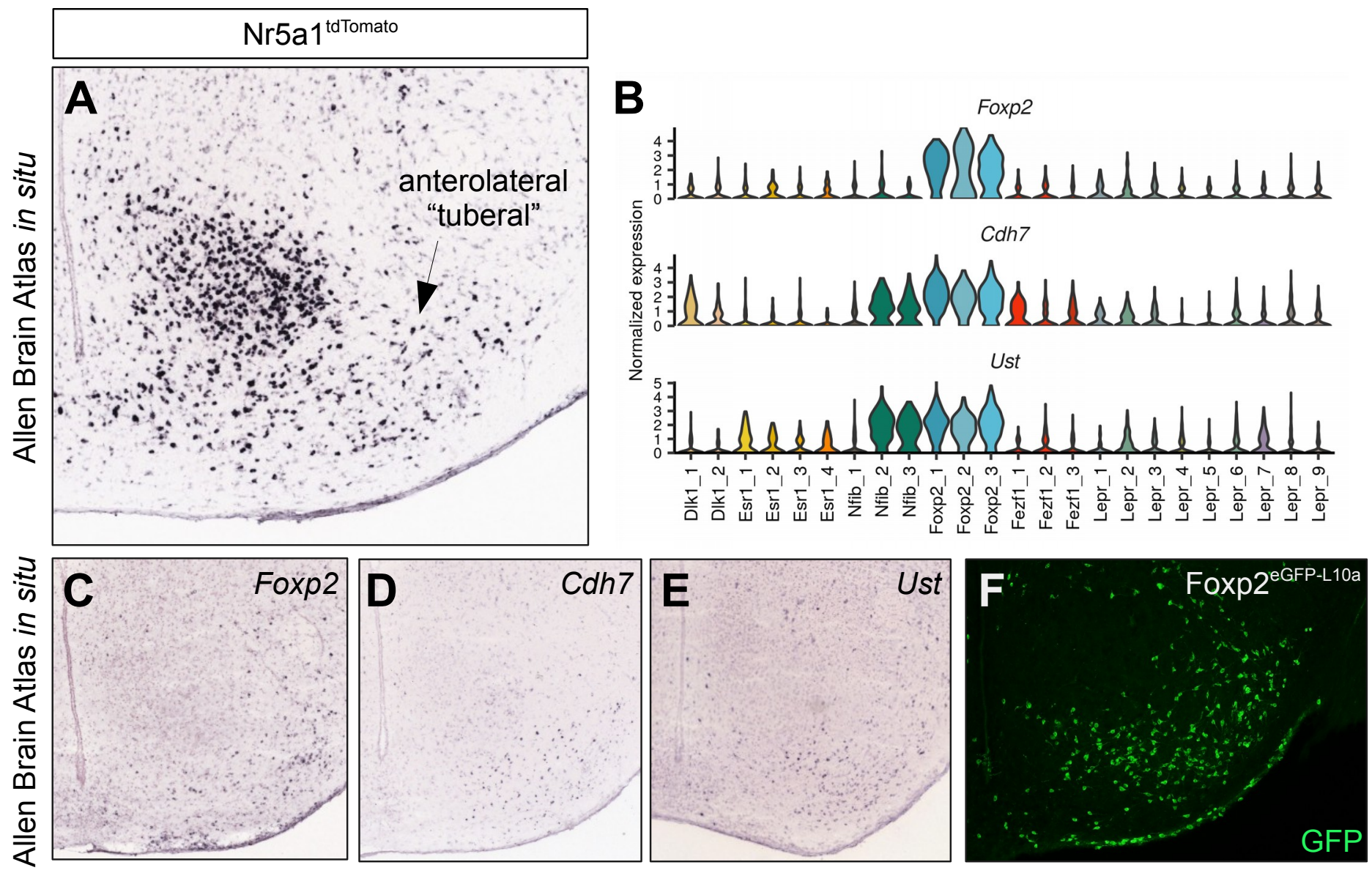

Figure S6: Foxp2 population localizes to anterolateral ("tuberal") compartment. (A) Allen Brain Atlas in situ for tdTomato in Nr5a1 ${ }^{\text {tdTomato }}$ mice (Nr5a1-Cre;R26 ${ }^{\text {LSL-tdTomato }}$ ) shows widespread expression outside of the core $\mathrm{VMH}$ in an area referred to as the tuberal nucleus. (B) The $\mathrm{VMH}^{\mathrm{Foxp2}}$ population is also marked by $C d h 7$ and Ust expression. (C-E) Allen Brain Atlas in situ images for (C) Foxp2, (D) Cdh7, and (E) Ust show expression in the tuberal VMH. (F) Representative image showing the distribution of GFP-IR (green) in Foxp2 ${ }^{\text {eGFP-L10a }}\left(\right.$ Foxp2 $\left.{ }^{\text {Cre/t; }} ; R_{26}{ }^{\text {eGFP-L10a }}\right)$ mice in the tuberal region. 


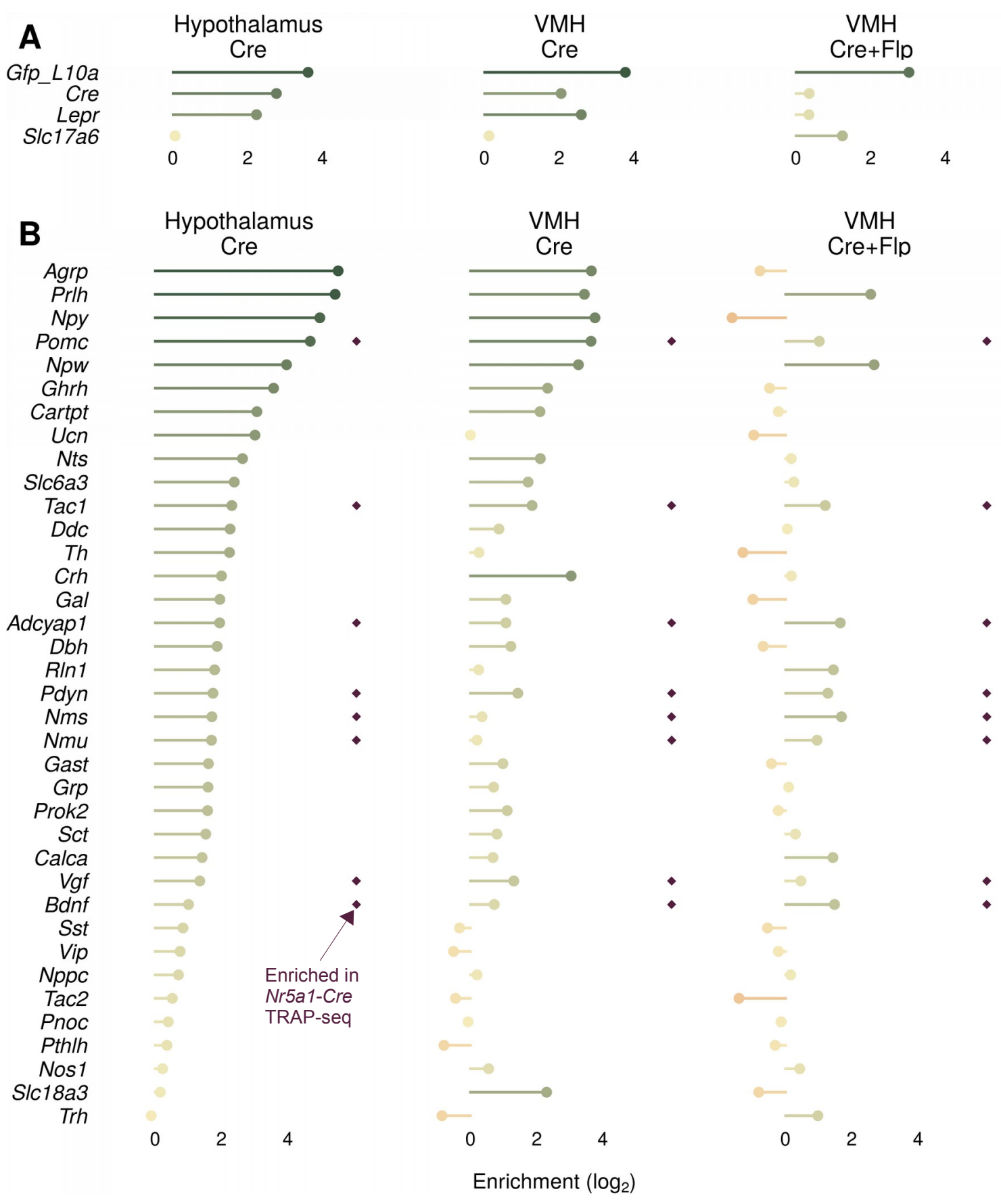

Figure S7: Comparison of different TRAP-seq approaches for identifying genes enriched in Lepr VMH cells. (A, B) Comparison of Lepr ${ }^{\text {EGFP-L10a }}\left(\right.$ Lepr $\left.^{\text {Cre }} ; R 26^{\text {LSLeGFP-L10a }}\right)$ of the whole hypothlalmus (Hypothalamus Cre), Lepr ${ }^{\text {eGFP-L10a }}$ with targeted dissection of the VMH (VMH Cre), and using the dual Flp- and Cre-dependent RCFL ${ }^{\text {eGFP-L10a }}$ with $L e p{ }^{r r e} ; S / c 17 a 6^{F / p o}$ mice (VMH Cre+FIp). (A) Enrichment of control genes in each dataset. (B) Enrichment of genes conferring neurochemical identity that are significantly enriched in any of the 3 datasets. Dark red diamonds signify genes that are significantly enriched in the Nr5a1 ${ }^{\text {GGFP-L10a }}$ TRAP-seq (presumptive VMH). 


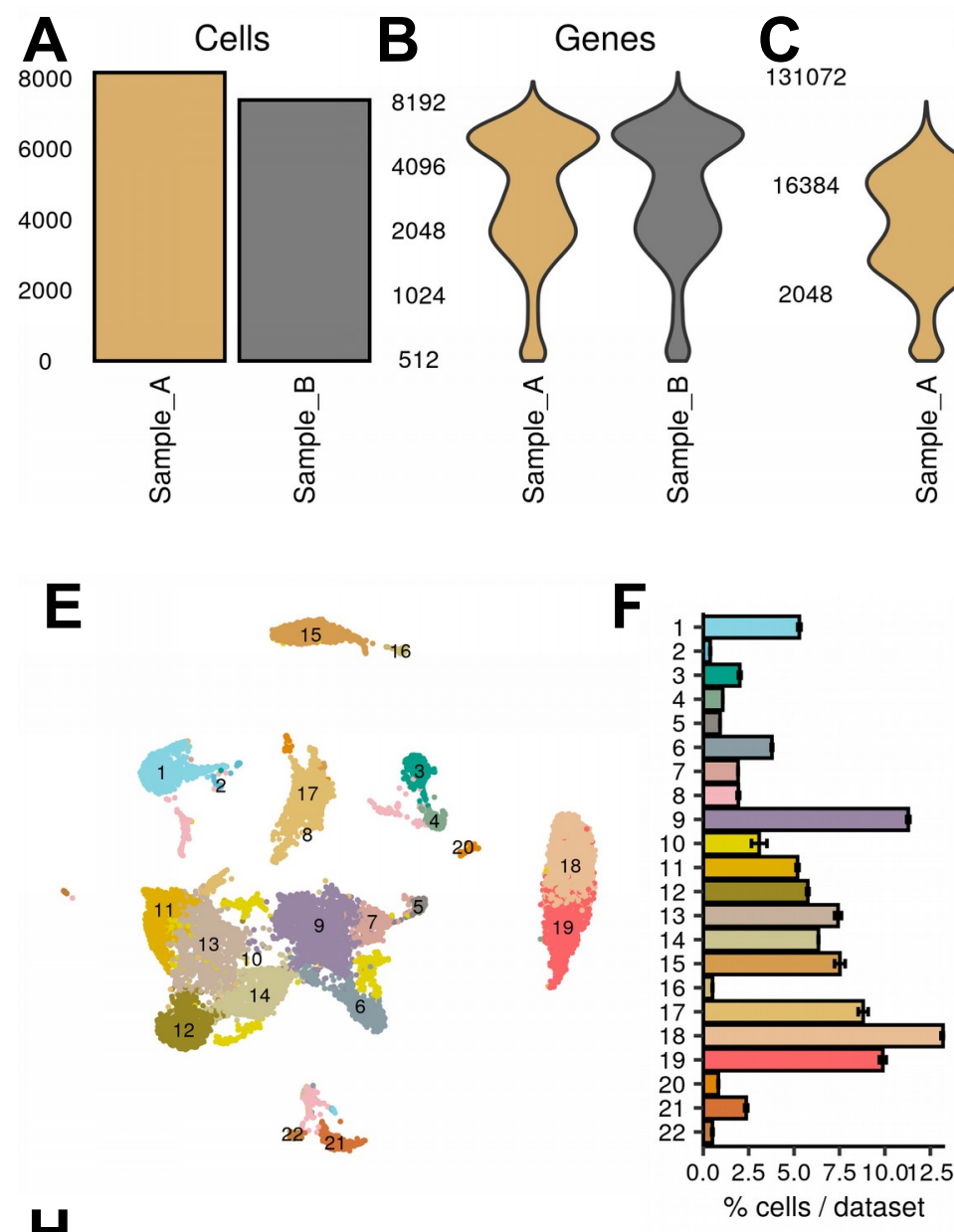

G
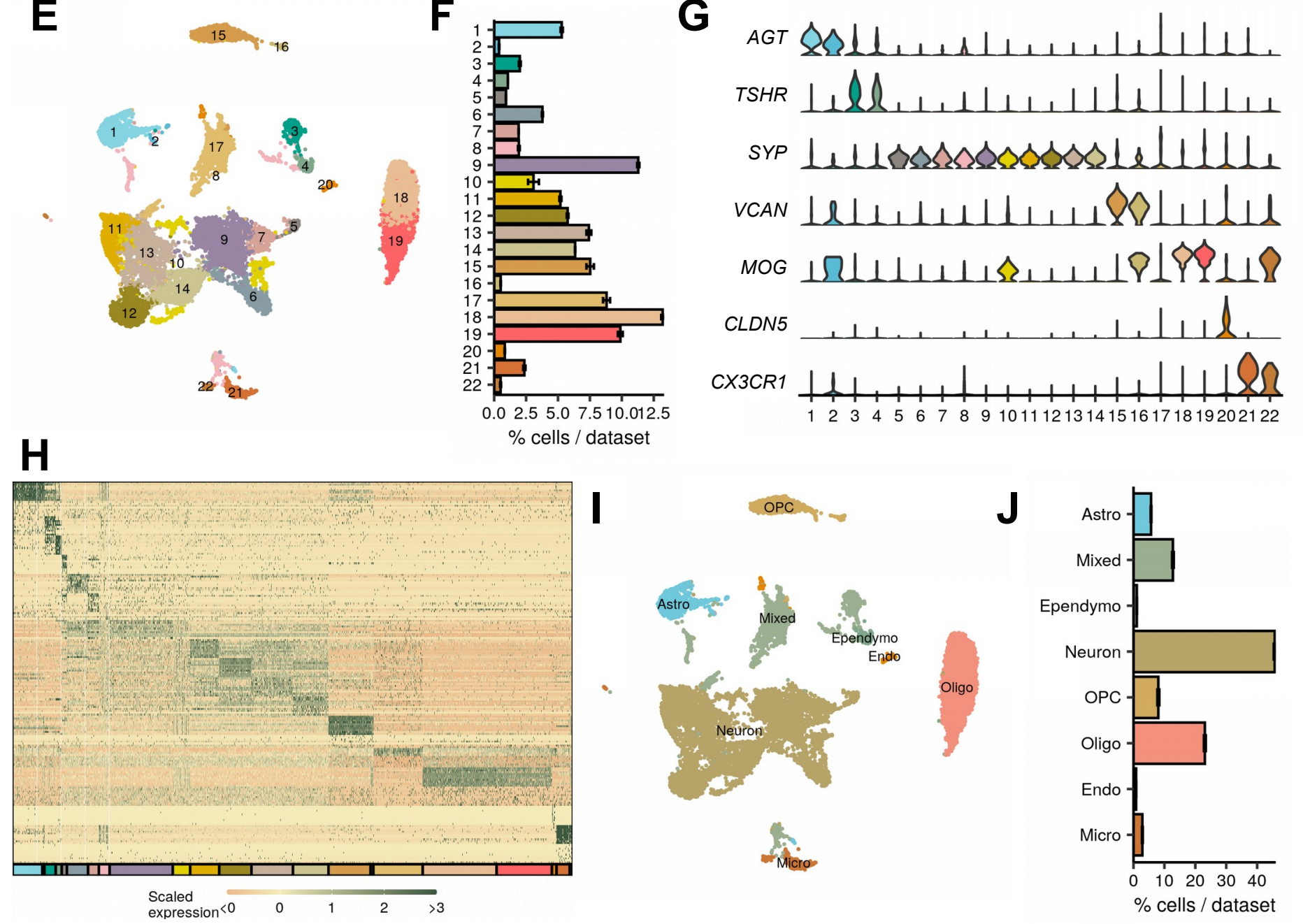

Figure S8: Macaque snRNA-seq identifies major CNS classes. (A-C) The number of $(\mathbf{A})$ cells, (B) genes, and (C) UMls detected per sample used in this study after quality control. (D-E) UMAP projection of all cells, colored by (D) sample and (E) cluster. (F) Average percent of cells in each cluster across all samples. (G) Representative marker genes for each of the major CNS cell types. (H) Expression profile of top 10 enriched genes for each cluster. (I) UMAP representation of cell type classification. (J) Quantification of cell classes per sample. 

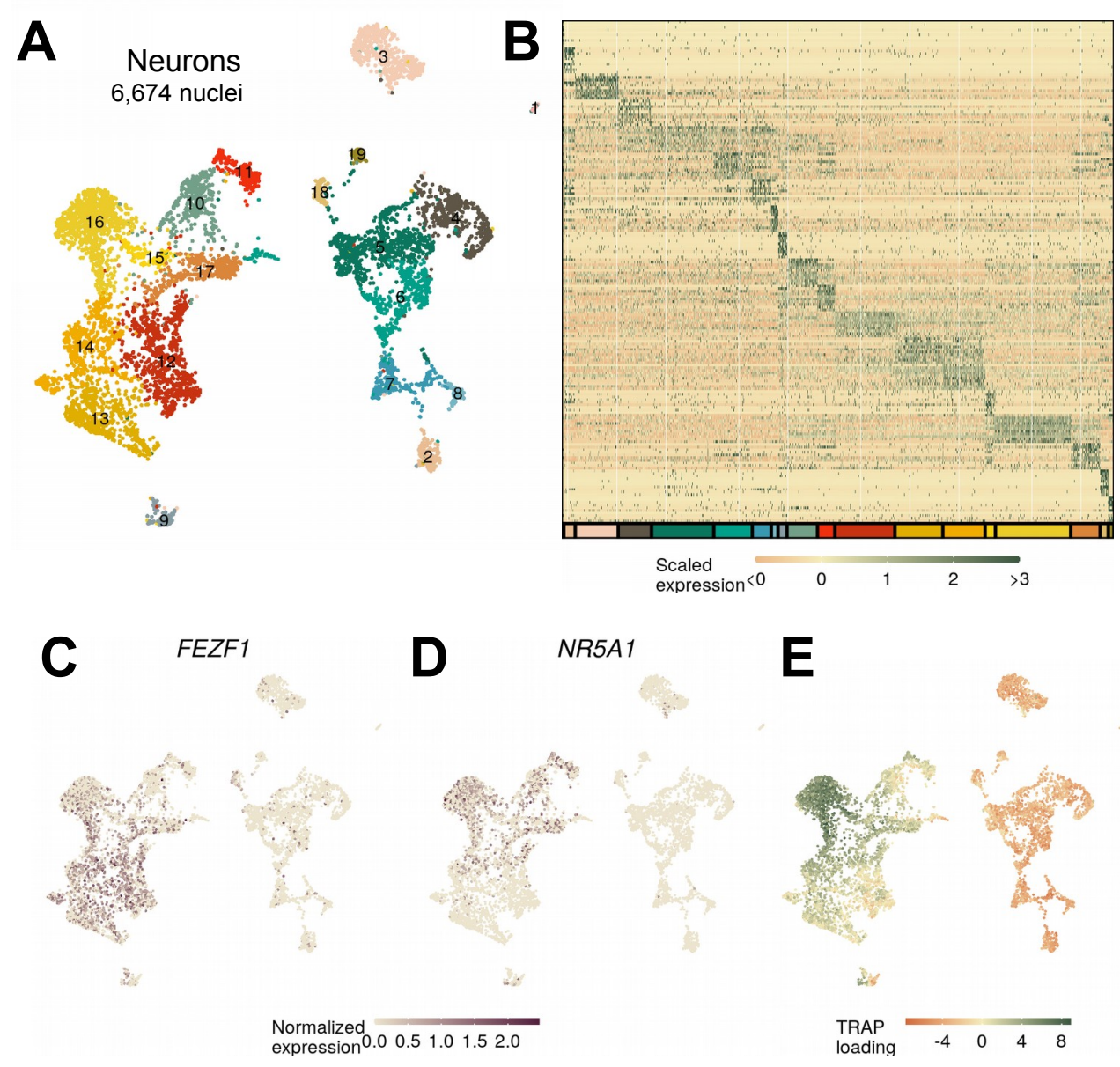

Figure S9: Identifying VMH neurons in macaque. (A) UMAP projection and labeling by cluster and (B) expression of top 10 genes for each macaque neuron cluster. (C) FEZF1 and (D) NR5A1 expression across the macaque neurons. (E) Loading on the top enriched mouse Nr5a1-Cre TRAP-seq genes. 


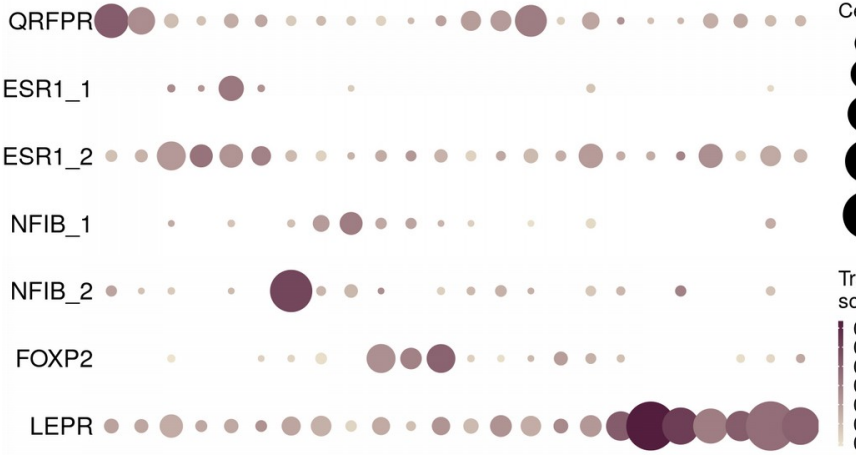

$-n_{1}-n_{1} m_{1} \nabla_{1}-n_{1} m_{1}-n_{1} m_{1}-n_{1} m_{1}-n_{1} m_{1} \nabla_{1} n_{1} \sigma_{1} r_{1} \infty_{1} \sigma_{1}$

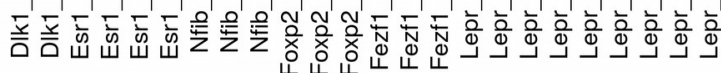

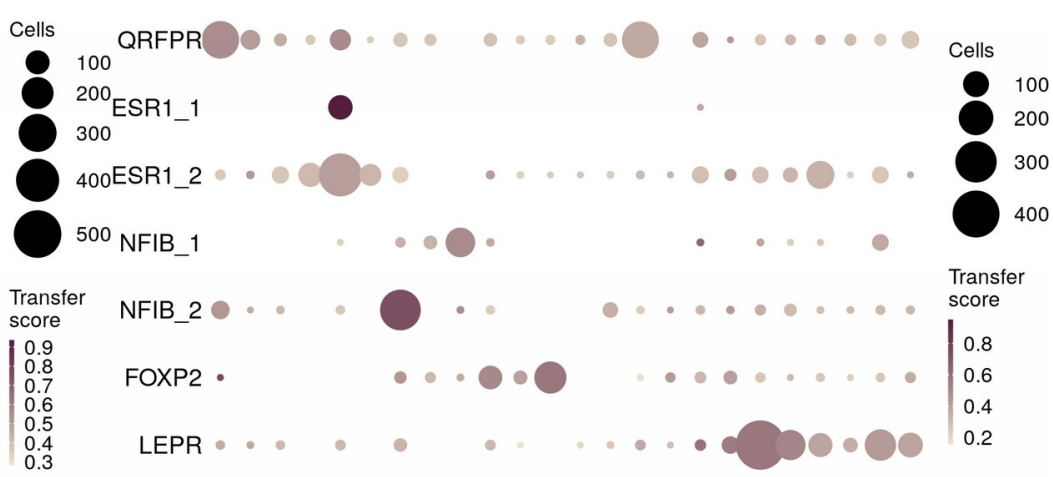

$-n-n m+-N m-n m-N m-n m+n \sigma \wedge m a$

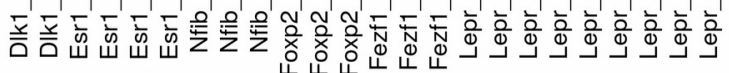

B

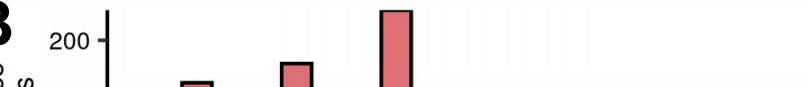

[ Mouse [ Common $[$ Macaque

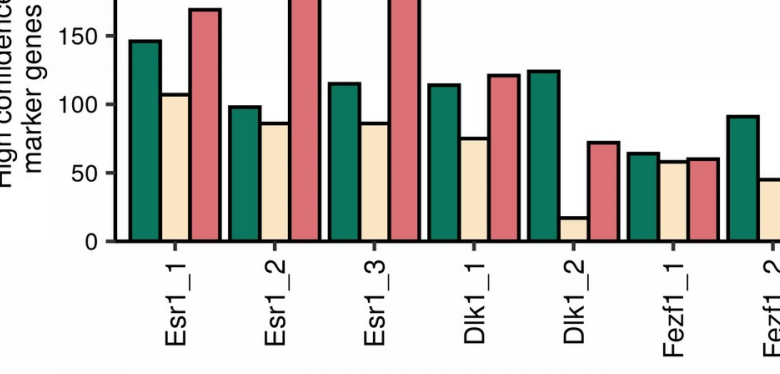

C

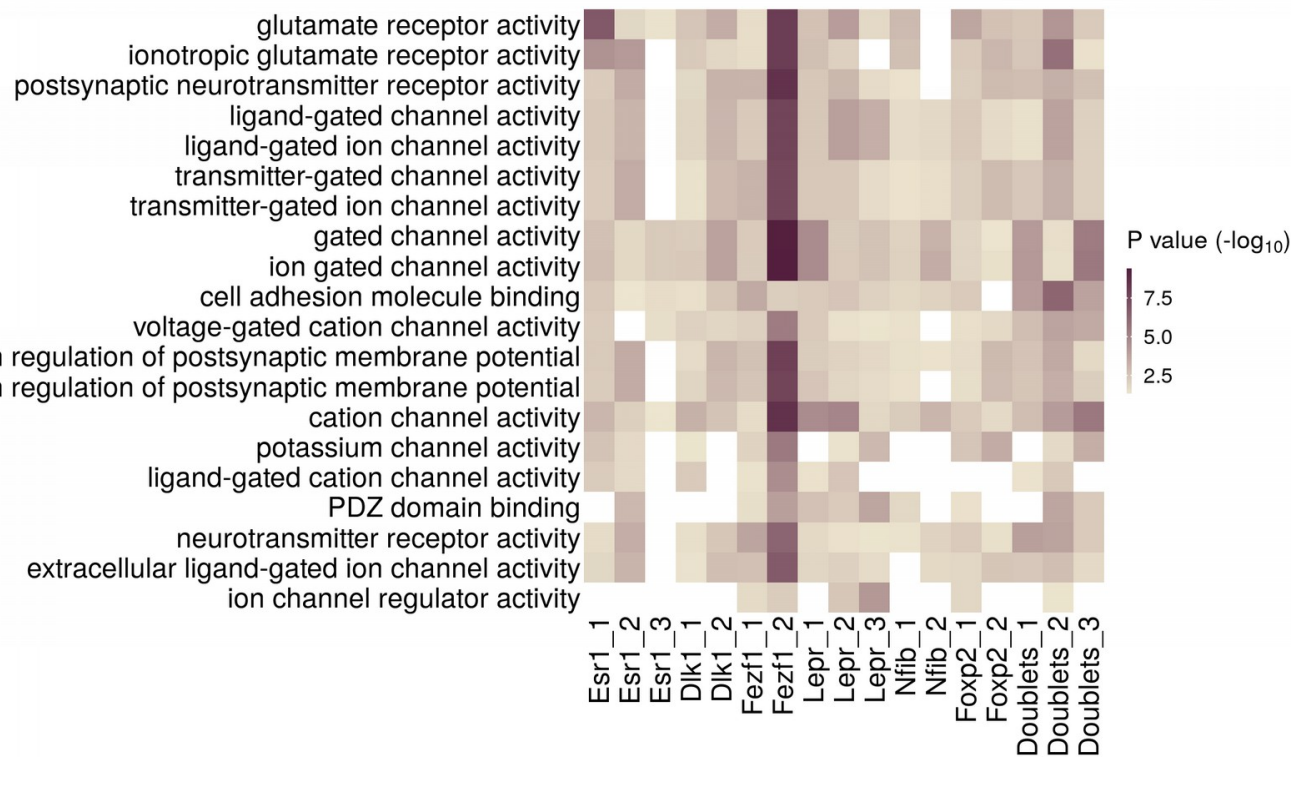

Figure S10: Similarities of mouse and macaque clusters. (A) Transferred cluster designations from mouse to macaque (and vice versa) using the Seurat CCA projection, colored by median transfer score with dot size corresponding to the number $\Phi$ cells from a given cluster transferred to each cluster. (B) Number of high confidence marker genes (CELLEX ES $\mu>0.5$ ) for each cluster and species. (C) Top GO terms associated with genes common between the species. (D) Pairwise expression correlation of 4866 orthologous marker genes between mouse and macaque classes. 

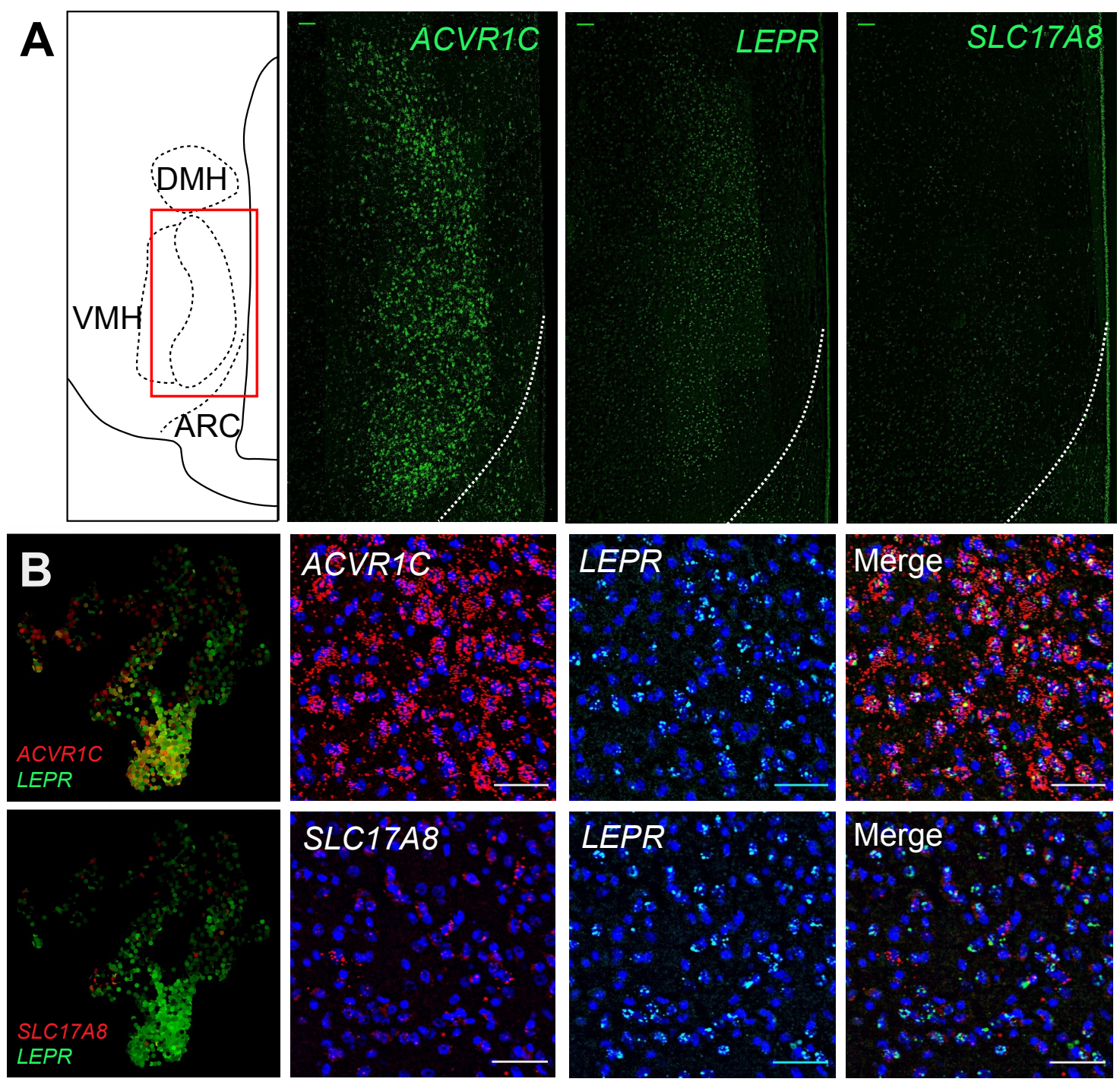

Figure S11: Cluster marker expression in macaque VMH. (A) (left column) Atlas image highlighting the region of interest and (other columns) representative fluorescent in situ hybridization images for ACVR1C, LEPR, and SLC17A8 (all in green). (B) (left column) Expression of marker genes in the macaque VMH neurons, projected into UMAP space. (other columns) Representative images showing DAPI (blue) and fluorescent in situ hybridization for ACVR1C (red, top) or SLC17A8 (red, bottom), LEPR (green), and merged images. 